\title{
Algorithmic trading for online portfolio selection under limited market liquidity
}

\author{
Youngmin $\mathrm{Ha}^{\mathrm{a}}$ and Hai Zhang ${ }^{\mathrm{b}}$ \\ ${ }^{\mathrm{a}}$ Adam Smith Business School, University of Glasgow, UK; ${ }^{\mathrm{b}}$ Department of Accounting \& \\ Finance, Strathclyde Business School, UK
}

(First draft: April 13, 2017; this draft: October 30, 2018)

\begin{abstract}
We propose an optimal intraday trading algorithm to reduce overall transaction costs through absorbing price shocks when an online portfolio selection (OPS) rebalances its portfolio. Having considered the real-time data of limit order books (LOB), the trading algorithm optimally splits a sizeable market order into a number of consecutive market orders to minimise the overall transaction costs, including both the market impact costs and the proportional transaction costs. The proposed trading algorithm, compatible to any OPS methods, optimises the number of intraday trades as well as finds an optimal intraday trading path. Backtesting results from the historical LOB data of NASDAQ-traded stocks show that the proposed trading algorithm significantly reduces the overall transaction costs in an environment of limited market liquidity.
\end{abstract}

Keywords: Algorithmic trading; Online portfolio selection; Market impact cost; Limit order book

JEL Classification: C61, C63, G11, G23

\section{Introduction}

Online portfolio selection (OPS) rebalances a portfolio in every period with the aim of maximising the portfolio's expected terminal wealth as a whole. OPS differs from the previous studies of prediction-based portfolio selection (Freitas et al. 2009, Otranto 2010, Ferreira and Santa-Clara 2011, DeMiguel et al. 2014, Palczewski et al. 2015), which i) forecast the expected values or covariance matrix of stock returns and ii) use the mean-variance optimisation. Most of the existing OPS methods (see Györfi and Vajda (2008), Kozat and Singer (2011) among others) consider only the proportional transaction costs (hereafter TCs). However, the liquidity risks or the market impact costs (MICs), a common feature of financial markets, has been ignored in OPS literature. Ha and Zhang (2018) did propose a more practical OPS method which considers both the proportional TCs and MICs, but none compatible optimal intraday trading algorithm has been developed in their framework to reduce the higher MICs under a limited market liquidity.

As Bertsimas and Lo (1998) pointed out" the demand for financial securities is not perfectly elastic, and the price impact of current trades, even small trades, can affect the course of future prices", then a big concern for existing OPS methods is its sizeable TCs when sending large market orders to rebalance a large-sized portfolio in each period. However, a large market order might not be executed if asset liquidity is limited at the rebalancing time. Even if it is executed, the transaction normally involves significantly high market impact costs (MICs henceforth) due to high volume trading, pushing the price up when buying the asset and pushing it down while

CONTACT Hai Zhang. Email: hai.zhang@strath.ac.uk 


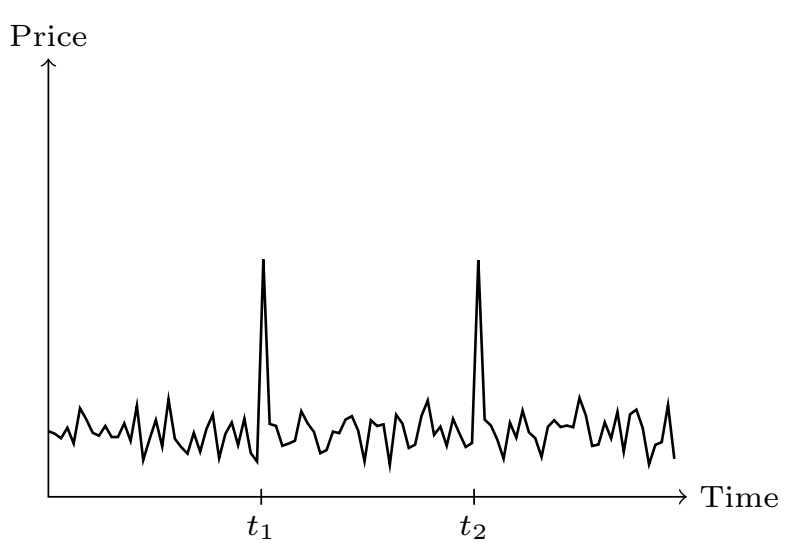

(a) Temporary impact.

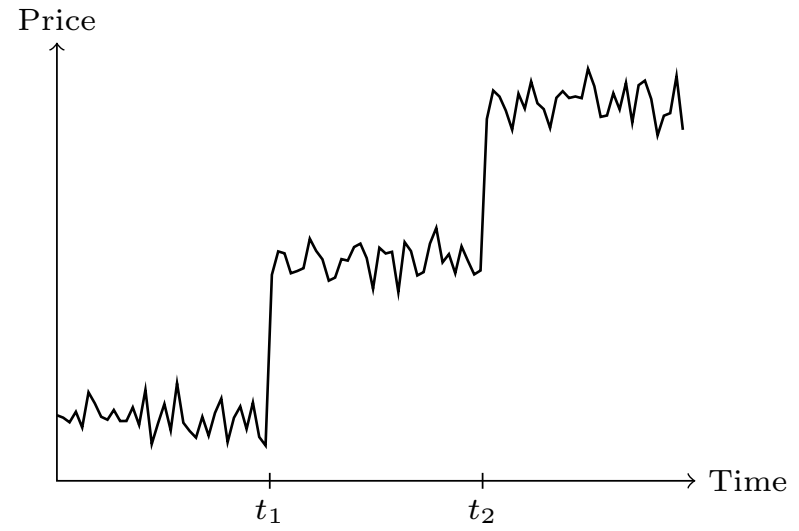

(b) Permanent impact.

Figure 1. Two types of stock price trajectory affected by two subsequent purchases at time $t_{1}$ and $t_{2}$ (similar graphs are in (Agliardi and Gençay 2014, p. 36)).

Table 1. A 5-level limit order book of Microsoft Corporation, traded on NASDAQ, on 21 Jun 2012 at 16:00:00 (downloaded from https://lobsterdata.com/info/DataSamples.php). Bid-ask spread is USD 0.01, and midpoint price is USD 30.135 .

\begin{tabular}{rcrr}
\hline & Level & Price (USD) & Volume (shares) \\
\hline \multirow{4}{*}{ Asks } & 5 & 30.18 & 110,006 \\
& 4 & 30.17 & 86,886 \\
& 3 & 30.16 & 65,399 \\
& 2 & 30.15 & 80,663 \\
& 1 & 30.14 & 16,600 \\
\hline \multirow{4}{*}{ Bids } & -1 & 30.13 & $-50,426$ \\
& -2 & 30.12 & $-83,306$ \\
& -3 & 30.11 & $-8,506$ \\
& -4 & 30.10 & $-43,838$ \\
& -5 & 30.09 & $-167,371$ \\
\hline
\end{tabular}

selling (Damodaran 2012, Chapter 5). Moreover, it might also cause a permanent rather temporary impact on asset prices ${ }^{1}$, thus making OPS strategies unprofitable. As the main assumption that portfolio rebalancing by OPS in the current period does not affect the stock prices in the next one is no longer valid should a permanent impact exist.

Existing trading algorithms might be considered for OPS to rebalance a large-sized portfolio, however, almost most of them are impractical as none of which considers the liquidity risk reflected by the limit order book (hereafter LOB; Table 1 provides an example of LOB). For example, Almgren and Chriss (2001) and Kissell et al. (2004) mathematically modelled market impact but did not consider LOB. Alfonsi et al. $(2008,2010)$ modelled the shape of LOB as a block or a continuous function rather than using LOB data. Guéant et al. (2012) used LOB data to calibrate the intensity parameters of trading execution, but they did not directly use LOB data for optimal trading.

The aim of this paper, therefore, is to develop an algorithmic trading strategy that splits a very large market order into a number of consecutive market orders to reduce the price shock, thus minimising the overall MICs when market liquidity is limited. This is straightforward as market usually absorbs shocks form these smaller slices, resulting in reduced MICs (Kissell et al. 2003, p. 196). To our knowledge, this paper is the first attempt to combine OPS with algorithmic trading under limited market liquidity. Propose an optimal intraday trading algorithm by considering real-time LOB data, compatible with any OPS method, available for any fund size, is the main contribution of the paper. Further, we run backtesting of the proposed intraday trading strategy

1 The permanent and temporary market impact have been defined and distinguished by Almgren and Chriss (2001) and see Figure 1 for an example of temporary and permanent market impact. 
using real-world historical NASDAQ LOB data.

The rest of this paper is organised as follows. Section 2 briefly reviews existing algorithmic trading strategies. Some key mathematical notations used in this paper are summarized in Section 3. Section 4 reviews a transaction cost factor (TCF) model considering MICs which serves as the foundation of the proposed intraday trading strategy. Section 5 develops an optimal intraday trading strategy for multi-asset portfolios, followed by an extensive backtesting in Section 6. Finally, Section 7 concludes.

\section{Literature review of algorithmic trading}

Algorithmic trading is the computerised execution of financial instruments following pre-specified rules and guidelines (Kissell 2013, p. 269), and it is classified by Kissell (2013, pp. 17-20) as follows:

(i) Arrival price algorithm that optimises a trading path in order to balance the trade-off between cost and risk at a user-specified level of risk aversion;

(ii) Implementation shortfall algorithm, which is similar to the arrival price algorithm, but incorporates real-time adaptation, i.e. the trading path of implementation shortfall algorithm is updated by real-time data on every intraday trade while that of the arrival price algorithm is determined before trading and does not change during an intraday trade;

(iii) Black box algorithm that searches for profitable opportunities and makes investment decisions based on market signals (e.g. asset prices and trading volume).

Based on the Kissell (2013)'s classification,we briefly review the aforementioned three trading algorithms in the following subsections.

\subsection{Arrival price algorithm}

Almgren and Chriss (2001) and Kissell et al. (2004) proposed an efficient frontier (akin to the Markowitz efficient frontier in the portfolio theory) in a two-dimensional plane whose axes are: the expected value of MIC arising from the temporary and permanent market impact (see Figure 1) and its variance, which comes from price volatility. Hence, the efficient frontier allows investors to choose their trading strategy for portfolio management with a user-specified parameter of risk aversion. The difference between the two methods is how to derive the equation of MIC. The former was derived from consecutive trades, whereas the latter was derived from an aggregate trade.

Alfonsi et al. (2008) suggested a trading strategy that splits a very large market order for a single-asset portfolio into a number of consecutive market orders to reduce expected overall market impact. The size of the individual orders is determined by a parameter of the resilience rate of a block-shaped LOB (it is assumed that an LOB consists of a continuous price distribution of orders with a constant height). However, the strategy does not consider the risk of price volatility as it assumes that traders are risk-neutral, which is different from the Almgren and Chriss (2001) and Kissell et al. (2004)'s trading strategy. Therefore, the Alfonsi et al.'s strategy minimises the expected value of MIC regardless of the risk.

Alfonsi et al. (2010) extended their previous trading strategy in LOBs of the constant function to one of a general shape function. They modelled discrete data of LOB as a continuous function of LOB density. Both strategies have the same optimal solution of intermediate orders: $\xi_{1}=\xi_{2}=\cdots=\xi_{N-1}$, where $\xi_{n}$ is the size of the market order placed at time $t_{n}$, and $t_{N}$ is the ending time of trading. However, the optimal initial market order $\xi_{0}$ for the generally-shaped LOBs is expressed as an implicit formula, while that for the block-shaped LOBs is expressed as an explicit formula. 


\subsection{Implementation shortfall algorithm}

A path-dependent or dynamic trading strategy by Lorenz (2008, Chapter 2-3), where the trading path is updated by real-time data, is superior in terms of generating a more efficient frontier to the path-independent or static trading strategy by Almgren and Chriss (2001), where the trading path is determined before trading starts. The superiority of the dynamic strategy comes from trading faster and reducing the risk of price volatility for the remaining time in the future if there was a windfall trading gain (i.e. lower trading cost) in the past.

Two other trading strategies as in Almgren and Chriss (2001), Guéant et al. (2012) respectively are similar in terms of liquidating a certain quantity of a single-asset portfolio within a given time horizon. However, they are different in terms of order types and optimisation methods. The former sends market orders by considering the trade-off between price risk and MICs, whereas the latter sends limit orders by considering both price risk and non-execution risk. Moreover, the Almgren and Chriss's strategy uses quadratic programming to construct an efficient frontier where the trade-off between price risk and MICs is binding, whereas the Guéant et al.'s strategy applies the Hamilton-Jacobi-Bellman equation to solve the stochastic control problem of optimal liquidation.

\subsection{Black box algorithm}

The following types of black box algorithm are reviewed:

- Pairs trading

Statistical arbitrage strategies based on cointegrated pairs of assets;

- High-frequency trading

Short-term trading strategies using sophisticated mathematics and high-speed computers;

- Artificial intelligence trading

Alpha generation strategies using artificial intelligence techniques.

2.3.1. Pairs trading. Avellaneda and Lee (2010) constructed a statistical arbitrage strategy of a market-neutral long-short portfolio. To be specific, they used pairs trading: long one dollar in a stock and short $\beta_{j}$ dollars in the $j$-th factor, where a multi-factor regression model decomposes a stock return $R$ into the sum of systematic components $\sum_{j=1}^{m} \beta_{j} F_{j}$ ( $m$ is the number of factors) and an idiosyncratic component $\tilde{R}$ :

$$
R=\sum_{j=1}^{m} \beta_{j} F_{j}+\tilde{R} .
$$

This method generates trading signals of buy, sell, or close of the long (short) position by using the mean-reverting property of the long-short portfolio's return.

Similar to Avellaneda and Lee (2010), Caldeira and Moura (2013) also considered a market-neutral strategy but used a different long-short portfolio. Avellaneda and Lee's strategy constructed either a pair of one stock and multiple exchange-traded funds or a pair of one stock and factors calculated from the principal component analysis while Caldeira and Moura constructed only two stocks as a pair. Moreover, if these two stocks selected are cointegrated, then it is possible to form a mean-reverting stationary process from a linear combination of stock $\mathrm{A}$ and $\mathrm{B}$.

2.3.2. High-frequency trading. A Markov chain model of the short-term dynamics of a LOB was proposed by Cont et al. (2010). To be specific, the volume of limit orders (see Table 1) is modelled as a Markov state, where a state transition occurs by a limit order, a market order, or a stop order. Furthermore, this model can be applied to high-frequency trading by making a short-term prediction of the mid-price and making a round-trip transaction. It enters a long position 
when the probability of the mid-price increasing is high, and it exits the position either when a profit is secured or when a loss of one tick is made.

A high-frequency arbitrage opportunity through an empirical analysis of the LOB resiliency of stocks traded on Shenzhen Stock Exchange was found (Xu et al. 2017). The analysis showed that buy (sell) market orders attract more buy (sell) limit orders especially i) when the bid-ask spread is one tick and ii) when the buy (sell) market order size is less than the best ask (bid) volume, which is the volume of LOB at level $1(-1)$ (see Table 1 ).

2.3.3. Artificial intelligence trading. Tan et al. (2011) explained how to detect stock cycles from historical stock prices. Based on the mean reverting property of stock prices they provided a reinforcement learning framework to trade on the cycles. Specifically, long positions are held after detecting troughs of stock cycles, and short positions are held after detecting peaks of stock cycles. In addition, a dynamic asset switching strategy was proposed to detect buying opportunities (troughs) of all assets in a portfolio.

Mousavi et al. (2014) proposed a multi-tree genetic programming model that i) extracts profitable trading rule bases for a multi-asset portfolio from historical data (daily closing price and transaction volume) and ii) updates the portfolio weights over time. Even though it generates a distinct decision rule for each stock, the rules for multiple stocks evolve simultaneously, and the correlations among multiple stocks are taken into account. In addition, the proposed model includes the risks and transaction costs to make the trading system more realistic.

Bendtsen and Peña (2016) developed a single-asset trading algorithm with either a long or closed position (i.e. no short selling) by using technical indicators such as moving average and relative strength index. Its goal is to generate buy or sell signals for trading a stock by learning and predicting the stock movement. A gated Bayesian network has been adopted to create a lower risk investment strategy compared with the buy-and-hold strategy. The network goes back and forth between the buy and sell phases and seeks opportunities to buy or sell shares.

Krauss et al. (2017) generated daily trading signals from lagged returns of stocks. They conducted nonparametric nonlinear regression between the lagged returns and one-day-ahead return. In particular, the following nonlinear regression methods were employed: deep neural networks, gradient-boosted trees, and random forests. After the regression, a daily portfolio of either going long for the stocks of higher expected returns or going short for lower expected return stocks has been constructed from the combined signals of the three methods.

\section{Notations}

The following notations are used in this paper:

- A lower case italic letter $x$ indicates a deterministic scalar value, while a capital italic letter $X$ indicates a random variable. A lower case italic bold letter $\boldsymbol{x}$ indicates a deterministic vector, while a capital italic bold letter $\boldsymbol{X}$ indicates a multivariate random variable (i.e. a random vector). A capital upright bold letter $\mathbf{X}$ denotes a deterministic matrix or a random matrix.

- $\mathbb{R}_{+}=\{x \in \mathbb{R} \mid x>0\}$ denotes positive real numbers.

- $\boldsymbol{b}_{n}=\left[\begin{array}{llll}b_{n}^{(1)} & b_{n}^{(2)} & \ldots & b_{n}^{(d)}\end{array}\right]^{\mathrm{T}}$ is a portfolio vector of $d$ risky assets (there is no risk-free asset in the portfolio) on the $n$-th day (see Figure 2), where $n \in\{1,2, \ldots\}, b_{n}^{(j)} \in 0 \cup \mathbb{R}_{+}$(i.e. neither short selling nor buying stocks on margin is permitted), and $\sum_{j=1}^{d} b_{n}^{(j)}=1$ (i.e. $b_{n}^{(j)}$ is the proportion of a portfolio invested in asset $j \in\{1,2, \ldots, d\}$ at the $n$-th day). Hence, $\boldsymbol{b}_{n} \in \Delta^{d-1}$, where $\Delta^{d-1}=\left\{\left[\begin{array}{llll}b^{(1)} & b^{(2)} & \ldots & b^{(d)}\end{array}\right]^{\mathrm{T}} \in \mathbb{R}^{d} \mid \sum_{j=1}^{d} b^{(j)}=1, b^{(j)} \geq 0\right\}$ is the standard $(d-1)$-simplex.

- $\boldsymbol{b}_{n, t} \in \Delta^{d-1}$ is an intraday portfolio vector at time $t$ after the end of the $n$-th day, where $t \in\{0,1, \ldots, \tau\}$, and $\tau$ is the number of intraday trades (see Figure 2; the portfolio rebalancing 

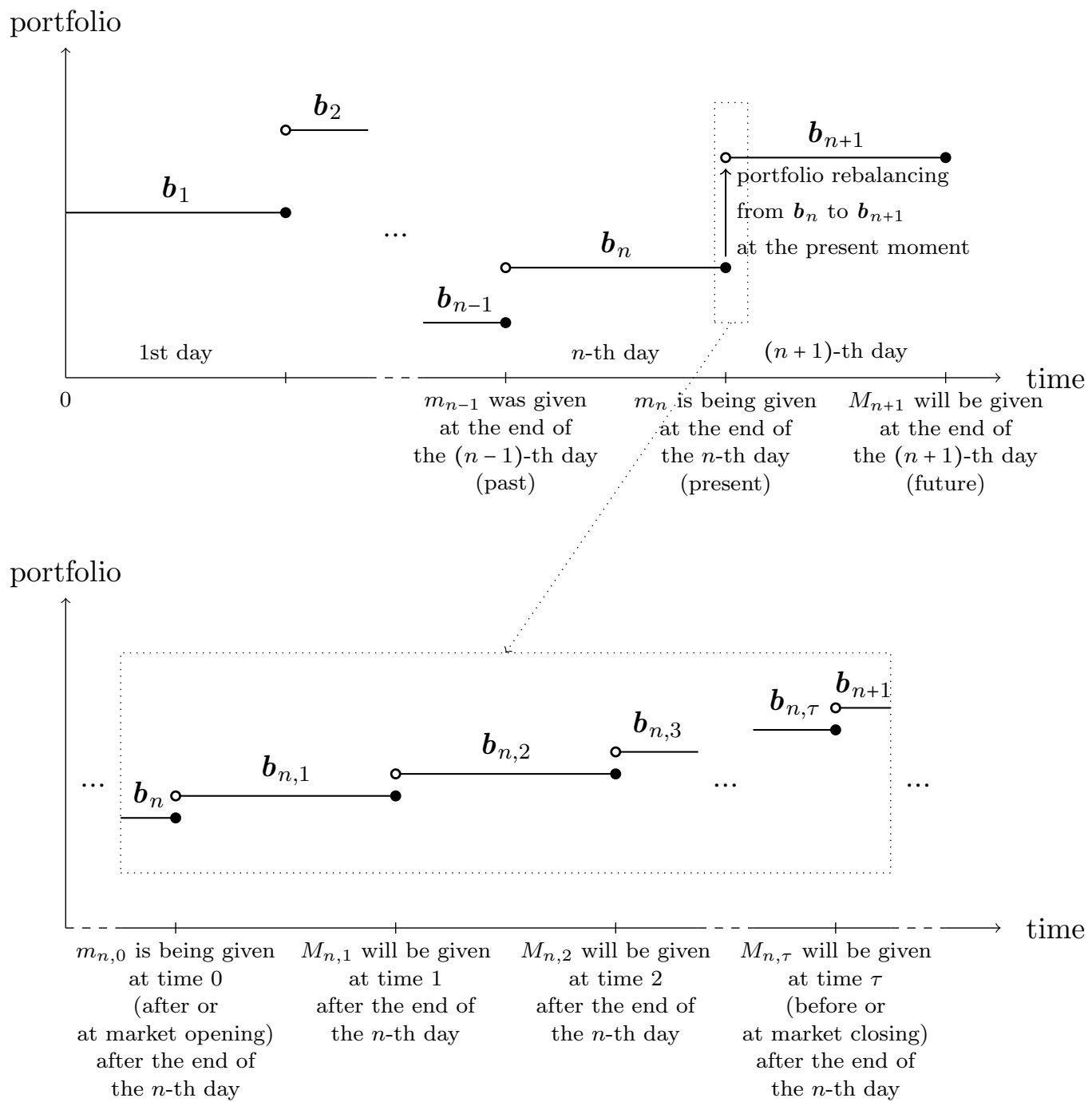

Figure 2. The timeline of intraday trading when the present moment is the end of $n$-th day. OPS rebalances a portfolio at the end of every trading day (a day ends at time 0, e.g. 9:30 a.m. or 10:00 a.m., not midnight, in this paper), and an algorithmic trading strategy cushions the shock by portfolio rebalancing from $\boldsymbol{b}_{n}$ to $\boldsymbol{b}_{n+1}$.

from $\boldsymbol{b}_{n, \tau}$ to $\boldsymbol{b}_{n+1}$ is not counted as an intraday trade).

- $\boldsymbol{b}_{1}=[1 / d 1 / d \ldots 1 / d]^{\mathrm{T}}$ is an initial portfolio vector.

- A deterministic value $m_{n}^{(j)}$ (if $n$ is a past or present day), or a random variable $M_{n}^{(j)}$ (if $n$ is a future day) is the mid-price of asset $j$ at the end of the $n$-th day.

- A deterministic value $m_{n, t}^{(j)}$ (if $n, t$ is in the past or at the present), or a random variable $M_{n, t}^{(j)}$ (if $n, t$ is in the future) is the mid-price of asset $j$ at time $t$ after the end of the $n$-th day. Technically, $m_{n, 0}^{(j)}=m_{n}^{(j)}$ and $M_{n, 0}^{(j)}=M_{n}^{(j)}$.

- A deterministic value $x_{n}^{(j)}=\frac{m_{n}^{(j)}}{m_{n-1}^{(j)}}$, or a random variable $X_{n}^{(j)}=\frac{M_{n}^{(j)}}{M_{n-1}^{(j)}}\left(\right.$ or $\left.X_{n}^{(j)}=\frac{M_{n}^{(j)}}{m_{n-1}^{(j)}}\right)$ is the relative price of asset $j$ for one day at the end of the $n$-th day.

- A deterministic vector $\boldsymbol{x}_{n}=\left[\begin{array}{lll}x_{n}^{(1)} & x_{n}^{(2)} \ldots x_{n}^{(d)}\end{array}\right]^{\mathrm{T}} \in \mathbb{R}_{+}^{d}$, or a multivariate random variable $\boldsymbol{X}_{n}=\left[\begin{array}{llll}X_{n}^{(1)} & X_{n}^{(2)} & \ldots & X_{n}^{(d)}\end{array}\right]^{\mathrm{T}} \in \mathbb{R}_{+}^{d}$ is the vector of the relative prices of all assets at the end of the $n$-th day $\left(\boldsymbol{x}_{n}\right.$ or $\boldsymbol{X}_{n}$ is called a market vector in this paper). 
- A deterministic value $x_{n, t}^{(j)}=\frac{m_{n, t}^{(j)}}{m_{n, t-1}^{(j)}}$, or a random variable $X_{n, t}^{(j)}=\frac{M_{n, t}^{(j)}}{M_{n, t-1}^{(j)}}\left(\right.$ or $\left.X_{n, t}^{(j)}=\frac{M_{n, t}^{(j)}}{m_{n, t-1}^{(j)}}\right)$ is the intraday relative price of asset $j$ between time $t-1$ and $t$ after the end of the $n$-th day, where $m_{n,-1} \stackrel{\text { def }}{=} m_{n-1, \tau}$, and $M_{n,-1} \stackrel{\text { def }}{=} M_{n-1, \tau}$.

- A deterministic vector $\boldsymbol{x}_{n, t}=\left[\begin{array}{llll}x_{n, t}^{(1)} & x_{n, t}^{(2)} & \ldots & x_{n, t}^{(d)}\end{array}\right]^{\mathrm{T}} \in \mathbb{R}_{+}^{d}$, or a multivariate random variable $\boldsymbol{X}_{n, t}=\left[\begin{array}{llll}X_{n, t}^{(1)} & X_{n, t}^{(2)} & \ldots & X_{n, t}^{(d)}\end{array}\right]^{\mathrm{T}} \in \mathbb{R}_{+}^{d}$ is the vector of the intraday relative prices of all assets, at time $t$ after the end of the $n$-th day $\left(\boldsymbol{x}_{n, t}\right.$ or $\boldsymbol{X}_{n, t}$ is called an intraday market vector in this paper).

\section{Market impact costs and transaction cost factor}

\subsection{Market impact costs (MICs) as a function of order size in a limit order book}

MIC is incurred when rebalancing a portfolio, and it can be written as a function of order volumes and prices in LOBs. The average MIC as a function of order size $q$ is defined as (Olsson 2005, Chapter 2.3)

$$
\begin{gathered}
\pi\left(q, m, p_{1}, p_{2}, \ldots, p_{-1}, p_{-2}, \ldots, v_{1}, v_{2}, \ldots, v_{-1}, v_{-2}, \ldots\right) \\
\stackrel{\text { def }\left|\bar{p}\left(q, m, p_{1}, p_{2}, \ldots, p_{-1}, p_{-2}, \ldots, v_{1}, v_{2}, \ldots, v_{-1}, v_{-2}, \ldots\right)-m\right|}{m},
\end{gathered}
$$

where $m=\frac{p_{-1}+p_{1}}{2}$ is the midpoint between the best bid and the best ask price, called mid-price. The average price per share for the order size $q$ is defined as

$$
\begin{gathered}
\bar{p}\left(q, m, p_{1}, p_{2}, \ldots, p_{-1}, p_{-2}, \ldots, v_{1}, v_{2}, \ldots, v_{-1}, v_{-2}, \ldots\right) \\
\stackrel{\text { def }}{=}\left\{\begin{array}{cl}
\frac{\sum_{i=k+1}^{-1} p_{i} v_{i}+p_{k}\left(q-\sum_{i=k+1}^{-1} v_{i}\right)}{q}, & \text { if } q<v_{-1} \\
p_{-1}, & \text { if } v_{-1} \leq q<0 \\
m, & \text { if } q=0 \\
p_{1}, & \text { if } 0<q \leq v_{1} \\
\frac{\sum_{i=1}^{k-1} p_{i} v_{i}+p_{k}\left(q-\sum_{i=1}^{k-1} v_{i}\right)}{q}, & \text { if } v_{1}<q
\end{array}\right.
\end{gathered}
$$

where positive (negative) $q$ means buying (selling) stocks, $p_{i}$ and $v_{i}$ with positive (negative) $i$ are the quoted ask (bid) price and volume at level $i$, respectively $\left(p_{i}\right.$ and $v_{i}$ correspond to the second and third column of Table 1 , respectively, where $\left.v_{i} \geq 0, v_{-i} \leq 0, \forall i \in\{1,2, \ldots\}\right)$, and the highest (lowest) trading level $k$ when $q>v_{1}\left(q<v_{-1}\right)$ is

$$
k=\left\{x \in \mathbb{Z} \mid x \geq 2, \sum_{i=1}^{x-1} v_{i}<q \leq \sum_{i=1}^{x} v_{i}\right\}
$$




$$
\left(k=\left\{x \in \mathbb{Z} \mid x \leq-2, \sum_{i=x}^{-1} v_{i} \leq q<\sum_{i=x+1}^{-1} v_{i}\right\}\right)
$$

i.e. $k$ represents the level in the order book where the $q$-th share would be executed.

\subsection{Transaction cost factor with both proportional transaction costs and market impact costs}

The net wealth at time $t$ after the end of the $n$-th day is a deterministic value $\nu_{n, t}$ if $n, t$ is in the past or at the present; while it is a random variable $N_{n, t}$ if $n, t$ is in the future (the notations of random variables are omitted in the subsequent expressions). The net wealth is defined as

$$
\nu_{n, t} \stackrel{\text { def }}{=} s_{n, t}-\gamma_{n, t}
$$

where $s_{n, t}$ is the gross wealth at time $t$ after the end of the $n$-th day, and $\gamma_{n, t}$ is transaction cost (TC) at time $t$ after the end of the $n$-th day. The gross wealth $s_{n, t}$ can be calculated from the previous net wealth $\nu_{n, t-1}$ :

$$
s_{n, t}=\nu_{n, t-1} \sum_{j=1}^{d} b_{n, t}^{(j)} x_{n, t}^{(j)}=\nu_{n, t-1}\left\langle\boldsymbol{b}_{n, t}, \boldsymbol{x}_{n, t}\right\rangle
$$

where $\nu_{n,-1} \stackrel{\text { def }}{=} \nu_{n-1, \tau}$, and $\langle\cdot, \cdot\rangle$ denotes the inner product. Transaction cost factor (TCF) at time $t$ after the end of the $n$-th day is defined as (Györfi and Vajda 2008)

$$
w_{n, t} \stackrel{\text { def }}{=} \frac{\nu_{n, t}}{s_{n, t}}
$$

where $w_{n, t} \in(0,1] \Leftrightarrow \gamma_{n, t} \in\left[0, s_{n, t}\right)$.

Let us calculate $w_{n, t}$ when rebalancing from $\boldsymbol{b}_{n, t}$ to $\boldsymbol{b}_{n, t+1}$. Followed by (Ha and Zhang 2018, Section 5.2-5.3), the gross wealth $s_{n, t}$, the sum of the net wealth $\nu_{n, t}$, the MICs, the purchase TCs, and the sale TCs, is given by

$$
s_{n, t}=\nu_{n, t}+\sum_{j=1}^{d}\left(\bar{p}\left(q_{n, t}^{(j)}\right)-m_{n, t}^{(j)}\right) q_{n, t}^{(j)}+c_{p} \sum_{j=1}^{d}\left(\bar{p}\left(q_{n, t}^{(j)}\right) q_{n, t}^{(j)}\right)^{+}+c_{s} \sum_{j=1}^{d}\left(-\bar{p}\left(q_{n, t}^{(j)}\right) q_{n, t}^{(j)}\right)^{+}
$$

where $c_{p}, c_{s} \in[0,1)$ denotes the rate of proportional TCs when purchasing and selling stocks respectively and $a^{+} \stackrel{\text { def }}{=} \max (0, a) .{ }^{2} q_{n, t}^{(j)}$ is an unknown order size of asset $j$ :

$$
q_{n, t}^{(j)}=\frac{b_{n, t+1}^{(j)} s_{n, t} w_{n, t}-b_{n, t}^{(j)} x_{n, t}^{(j)} \nu_{n, t-1}}{m_{n, t}^{(j)}} ;
$$

and $b_{n, \tau+1}^{(j)} \stackrel{\text { def }}{=} b_{n+1}^{(j)}$. Equation (8) can be simplified, by the property of $a^{+}=a+(-a)^{+}$, as

$$
s_{n, t}=\nu_{n, t}+\sum_{j=1}^{d}\left(\left(1-c_{s}\right) \bar{p}\left(q_{n, t}^{(j)}\right)-m_{n, t}^{(j)}\right) q_{n, t}^{(j)}+\left(c_{p}+c_{s}\right) \sum_{j=1}^{d}\left(\bar{p}\left(q_{n, t}^{(j)}\right) q_{n, t}^{(j)}\right)^{+}
$$

\footnotetext{
2 Some arguments of Equation (3), $m, p_{1}, p_{2}, \ldots, p_{-1}, p_{-2}, \ldots, v_{1}, v_{2}, \ldots, v_{-1}, v_{-2}, \ldots$ of asset $j$, are omitted in Equation (8) and the subsequent expressions for notational simplicity
} 
and this can be rewritten, using Equation (7), as

$$
w_{n, t}=1-\frac{\sum_{j=1}^{d}\left(\left(1-c_{s}\right) \bar{p}\left(q_{n, t}^{(j)}\right)-m_{n, t}^{(j)}\right) q_{n, t}^{(j)}+\left(c_{p}+c_{s}\right) \sum_{j=1}^{d}\left(\bar{p}\left(q_{n, t}^{(j)}\right) q_{n, t}^{(j)}\right)^{+}}{s_{n, t}},
$$

where $w_{0, t} \stackrel{\text { def }}{=} 1, \forall t \in\{0,1, \ldots, \tau\}$ (i.e. there are no TCs between time 0 and $\tau$ on the 0 -th day). Equation (9) and (11) are solvable by using a root-finding algorithm, where $w_{n, t}=w\left(\boldsymbol{b}_{n, t}, \boldsymbol{b}_{n, t+1}, \boldsymbol{x}_{n, t}, \nu_{n, t-1}\right)$ is an unknown variable, $c_{p}$ and $c_{s}$ are omitted here for notational simplicity.

\section{Proposed method of optimal intraday trading}

This section explains:

(i) how to calculate the expected value of the gross wealth of tomorrow in the case of no intraday trading (i.e. $\tau=0$ );

(ii) how to obtain an optimal trading path when rebalancing a portfolio from $\boldsymbol{b}_{n}$ to $\boldsymbol{b}_{n+1}$, given the number of intraday trades $\tau \geq 1$ (see Figure 2);

(iii) how to calculate the optimal number of intraday trades $\tau^{*}$;

(iv) and how to consider real-time LOB data for optimal intraday trading.

The following assumptions are made for the simplicity of the proposed method:

Assumption 5.1 Asset prices follow the multi-dimensional Brownian motion with zero drift (forecasting expected returns is not performed in this paper);

- hence, the increments of asset prices are jointly normally distributed;

- and the increments of asset prices are mutually independent for different assets $j \neq j^{\prime}$ or different trading times $t \neq t^{\prime}$.

Assumption 5.2 LOB at time $t^{\prime} \in\{t+1, t+2, \ldots, \tau\}$ is the same as LOB at time $t$ on the same day.

\subsection{No intraday trading $(\tau=0)$}

Suppose that a filtered probability space $\left(\Omega, \mathcal{F},\left\{\mathcal{F}_{n}\right\}_{n \in\{1,2, \ldots\}}, \mathbb{P}\right)$ is given, where $\mathcal{F}_{n}$ denotes the natural filtration of the process $\left\{\boldsymbol{X}_{n}\right\}_{n \in\{1,2, \ldots\}}$ up to day $n$. If no intraday trading occurs, the conditional expected value of the gross wealth at the end of the $(n+1)$-th day, given the past observations $\boldsymbol{x}_{1}, \boldsymbol{x}_{2}, \ldots, \boldsymbol{x}_{n}$, and using Equation (6) and (7), is given by

$$
\begin{aligned}
\mathbb{E}\left[S_{n+1} \mid \mathcal{F}_{n}\right] & =\mathbb{E}\left[N_{n}\left\langle\boldsymbol{b}_{n+1}, \boldsymbol{X}_{n+1}\right\rangle \mid \mathcal{F}_{n}\right] \\
& =\mathbb{E}\left[S_{n} W_{n}\left\langle\boldsymbol{b}_{n+1}, \boldsymbol{X}_{n+1}\right\rangle \mid \mathcal{F}_{n}\right] \\
& =\mathbb{E}\left[S_{n} W_{n} \mid \mathcal{F}_{n}\right] \mathbb{E}\left[\left\langle\boldsymbol{b}_{n+1}, \boldsymbol{X}_{n+1}\right\rangle \mid \mathcal{F}_{n}\right] \\
& =s_{n} w_{n} \mathbb{E}\left[\left\langle\boldsymbol{b}_{n+1}, \boldsymbol{X}_{n+1}\right\rangle\right],
\end{aligned}
$$

where

- $N_{n}$ is stochastic net wealth at the end of the $n$-th day,

- $S_{n}=s_{0} \prod_{i=1}^{n}\left\langle\boldsymbol{b}_{i}, \boldsymbol{X}_{i}\right\rangle$ is stochastic gross wealth at the end of the $n$-th day with an initial wealth $s_{0}$, 
- $W_{n}=w\left(\boldsymbol{b}_{n}, \boldsymbol{b}_{n+1}, \boldsymbol{X}_{n}, N_{n-1}\right)$ is stochastic TCF at the end of the $n$-th day $\left(S_{n} W_{n}\right.$ and $\left\langle\boldsymbol{b}_{n+1}, \boldsymbol{X}_{n+1}\right\rangle$ are mutually independent by Assumption 5.1 that $\boldsymbol{X}_{n}$ and $\boldsymbol{X}_{n+1}$ are mutually independent),

- $s_{n}=s_{0} \prod_{i=1}^{n}\left\langle\boldsymbol{b}_{i}, \boldsymbol{x}_{i}\right\rangle$ is deterministic gross wealth at the end of the $n$-th day,

- $w_{n}=w\left(\boldsymbol{b}_{n}, \boldsymbol{b}_{n+1}, \boldsymbol{x}_{n}, \nu_{n-1}\right)$ is deterministic TCF at the end of the $n$-th day $\left(S_{n} W_{n}\right.$ is converted to $s_{n} w_{n}$ by the conditional expectation given $\mathcal{F}_{n}$ ),

- and the market vector $\boldsymbol{X}_{n+1}$ is jointly normally distributed with the mean vector of all 1's $\left[\begin{array}{llll}1 & 1 & \ldots & 1\end{array}\right]^{\mathrm{T}}$.

By Assumption 5.1, Equation (12) can be further simplified, and the price change $\boldsymbol{M}_{n+1}-\boldsymbol{m}_{n}$ follows the multi-dimensional Brownian motion with zero drift:

$$
\left(\boldsymbol{M}_{n+1}-\boldsymbol{m}_{n}\right) \sim \mathcal{N}\left(\boldsymbol{O}, \boldsymbol{\Sigma}_{n}\right),
$$

where $\boldsymbol{M}_{n+1}=\left[\begin{array}{llll}M_{n+1}^{(1)} & M_{n+1}^{(2)} & \ldots & M_{n+1}^{(d)}\end{array}\right]^{\mathrm{T}}$ is the random mid-price vector at the end of the $(n+1)$-th day, $\boldsymbol{m}_{n}=\left[m_{n}^{(1)} m_{n}^{(2)} \ldots m_{n}^{(d)}\right]^{\mathrm{T}}$ is the deterministic mid-price vector at the end of the $n$-th day, $\boldsymbol{O}$ denotes the all-zero vector, and $\boldsymbol{\Sigma}_{n}$ is the covariance matrix of price changes between the end of the $n$-th day and the end of the $(n+1)$-th day. Hence, $\boldsymbol{M}_{n+1}$ is jointly normally distributed as

$$
\boldsymbol{M}_{n+1} \sim \mathcal{N}\left(\boldsymbol{m}_{n}, \boldsymbol{\Sigma}_{n}\right) .
$$

Also, as $\boldsymbol{X}_{n+1}$ can be calculated as

$$
\boldsymbol{X}_{n+1}=\mathbf{D}^{-1} \boldsymbol{M}_{n+1},
$$

where $\mathbf{D}=\operatorname{diag}\left(\boldsymbol{m}_{n}\right)$, it is jointly normally distributed as

$$
\boldsymbol{X}_{n+1} \sim \mathcal{N}\left(1, \mathbf{D}^{-1} \boldsymbol{\Sigma}_{n} \mathbf{D}^{-1}\right) .
$$

Consequently, $\mathbb{E}\left[S_{n+1} \mid \mathcal{F}_{n}\right]$ in Equation (12) can be simplified as

$$
\begin{aligned}
\mathbb{E}\left[S_{n+1} \mid \mathcal{F}_{n}\right] & =s_{n} w_{n} \mathbb{E}\left[\left\langle\boldsymbol{b}_{n+1}, \boldsymbol{X}_{n+1}\right\rangle\right] \\
& =s_{n} w_{n} \mathbb{E}\left[b_{n+1}^{(1)} X_{n+1}^{(1)}+b_{n+1}^{(2)} X_{n+1}^{(2)}+\cdots+b_{n+1}^{(d)} X_{n+1}^{(d)}\right] \\
& =s_{n} w_{n}\left(b_{n+1}^{(1)} \mathbb{E}\left[X_{n+1}^{(1)}\right]+b_{n+1}^{(2)} \mathbb{E}\left[X_{n+1}^{(2)}\right]+\cdots+b_{n+1}^{(d)} \mathbb{E}\left[X_{n+1}^{(d)}\right]\right) \\
& =s_{n} w_{n} \sum_{j=1}^{d} b_{n+1}^{(j)} \\
& =s_{n} w_{n} .
\end{aligned}
$$

\subsection{Single intraday trade $(\tau=1)$}

If there is noly one intraday trading per trading day, then the conditional expected value of the gross wealth at the end of the $(n+1)$-th day, given the past observations $\boldsymbol{x}_{1}, \boldsymbol{x}_{2}, \ldots, \boldsymbol{x}_{n}$, and using Equation (6), (7), and (12), is given by

$$
\mathbb{E}\left[S_{n+1} \mid \mathcal{F}_{n}\right]=s_{n} w_{n} \mathbb{E}\left[\left\langle\boldsymbol{b}_{n, 1}, \boldsymbol{X}_{n, 1}\right\rangle W_{n, 1}\left\langle\boldsymbol{b}_{n+1}, \boldsymbol{X}_{n+1,0}\right\rangle\right],
$$

where 
- $w_{n}=w\left(\boldsymbol{b}_{n}, \boldsymbol{b}_{n, 1}, \boldsymbol{x}_{n, 0}, \nu_{n-1,1}\right)$ ( $w_{n}$ is TCF at the end of the $n$-th day when rebalancing a portfolio from $\boldsymbol{b}_{n}$ to $\left.\boldsymbol{b}_{n, 1}\right)$,

$\circ \boldsymbol{x}_{n, 0}=\left[\frac{m_{n}^{(1)}}{m_{n-1,1}^{(1)}} \frac{m_{n}^{(2)}}{m_{n-1,1}^{(2)}} \ldots \frac{m_{n}^{(d)}}{m_{n-1,1}^{(d)}}\right]^{\mathrm{T}}\left(\boldsymbol{x}_{n, 0}\right.$ is not equivalent to $\boldsymbol{x}_{n}=\left[\frac{m_{n}^{(1)}}{m_{n-1}^{(1)}} \frac{m_{n}^{(2)}}{m_{n-1}^{(2)}} \ldots \frac{m_{n}^{(d)}}{m_{n-1}^{(d)}}\right]^{\mathrm{T}}$ even though $m_{n, 0}^{(j)}$ is equivalent to $\left.m_{n}^{(j)}\right)$,

- $\boldsymbol{X}_{n, 1}=\left[\frac{M_{n, 1}^{(1)}}{m_{n}^{(1)}} \frac{M_{n, 1}^{(2)}}{m_{n}^{(2)}} \cdots \frac{M_{n, 1}^{(d)}}{m_{n}^{(d)}}\right]^{\mathrm{T}}$,

- $W_{n, 1}=w\left(\boldsymbol{b}_{n, 1}, \boldsymbol{b}_{n+1}, \boldsymbol{X}_{n, 1}, s_{n} w_{n}\right)$,

- and $\quad \boldsymbol{X}_{n+1,0}=\left[\frac{M_{n+1}^{(1)}}{M_{n, 1}^{(1)}} \frac{M_{n+1}^{(2)}}{M_{n, 1}^{(2)}} \cdots \frac{M_{n+1}^{(d)}}{M_{n, 1}^{(d)}}\right]^{\mathrm{T}} \quad\left(\boldsymbol{X}_{n+1,0} \quad\right.$ is $\quad$ not $\quad$ equivalent to $\boldsymbol{X}_{n+1}=\left[\frac{M_{n+1}^{(1)}}{m_{n}^{(1)}} \frac{M_{n+1}^{(2)}}{m_{n}^{(2)}} \ldots \frac{M_{n+1}^{(d)}}{m_{n}^{(d)}}\right]^{\mathrm{T}}$ even though $M_{n+1,0}^{(j)}$ is equivalent to $\left.M_{n+1}^{(j)}\right)$.

Of course, the MIC function in Equation (2) at time 1 after the end of the $n$-th day to calculate $W_{n, 1}$ is stochastic as LOBs are continuously updated by other investors between time 0 and 1 . However, the random variables $P_{i}^{(j)}, V_{i}^{(j)}, \forall i, j$ in LOBs at time 1 are omitted in Equation (18) for the simple expression, where $P_{i}^{(j)}\left(V_{i}^{(j)}\right)$ is a random variable of the quoted price (volume) of asset $j$ at level $i$ in LOBs. This corresponds to Assumption 5.2.

By Assumption 5.1 that the two random vectors $\boldsymbol{X}_{n, 1}, \boldsymbol{X}_{n+1,0}$ are mutually independent, Equation (18) can be rewritten again as

$$
\mathbb{E}\left[S_{n+1} \mid \mathcal{F}_{n}\right]=s_{n} w_{n} \mathbb{E}\left[\left\langle\boldsymbol{b}_{n, 1}, \boldsymbol{X}_{n, 1}\right\rangle W_{n, 1}\right] \mathbb{E}\left[\left\langle\boldsymbol{b}_{n+1}, \boldsymbol{X}_{n+1,0}\right\rangle\right],
$$

but $\left\langle\boldsymbol{b}_{n, 1}, \boldsymbol{X}_{n, 1}\right\rangle$ and $W_{n, 1}$ are mutually dependent as $W_{n, 1}$ is a function of $\boldsymbol{X}_{n, 1}$. Finally, by the property of $\mathbb{E}\left[\left\langle\boldsymbol{b}_{n+1}, \boldsymbol{X}_{n+1,0}\right\rangle\right]=1$, as proved in Equation (17), $\mathbb{E}\left[S_{n+1} \mid \mathcal{F}_{n}\right]$ in Equation (18) can be simplified as

$$
\mathbb{E}\left[S_{n+1} \mid \mathcal{F}_{n}\right]=s_{n} w_{n} \mathbb{E}\left[\left\langle\boldsymbol{b}_{n, 1}, \boldsymbol{X}_{n, 1}\right\rangle W_{n, 1}\right]
$$

Our goal is to find the optimal portfolio vector $\boldsymbol{b}_{n, 1}^{*}$ that maximises $\mathbb{E}\left[S_{n+1} \mid \mathcal{F}_{n}\right]$, and this is a stochastic programming problem defined as follows:

$$
\begin{aligned}
\boldsymbol{b}_{n, 1}^{*} & \underset{\boldsymbol{b}_{n, 1} \in \Delta^{d-1}}{\arg \max } \mathbb{E}\left[S_{n+1} \mid \mathcal{F}_{n}\right] \\
= & \underset{\boldsymbol{b}_{n, 1} \in \Delta^{d-1}}{\arg \max } s_{n} w_{n} \mathbb{E}\left[\left\langle\boldsymbol{b}_{n, 1}, \boldsymbol{X}_{n, 1}\right\rangle W_{n, 1}\right] \\
= & \underset{\boldsymbol{b}_{n, 1} \in \Delta^{d-1}}{\arg \max } w_{n} \mathbb{E}\left[\left\langle\boldsymbol{b}_{n, 1}, \boldsymbol{X}_{n, 1}\right\rangle W_{n, 1}\right] \\
= & \underset{\boldsymbol{b}_{n, 1} \in \Delta^{d-1}}{\arg \max } w_{n} \int_{\boldsymbol{x}_{n, 1} \in \mathbb{R}_{+}^{d}}\left\langle\boldsymbol{b}_{n, 1}, \boldsymbol{x}_{n, 1}\right\rangle w_{n, 1} f\left(\boldsymbol{x}_{n, 1}\right) d \boldsymbol{x}_{n, 1},
\end{aligned}
$$

where $w_{n, 1}=w\left(\boldsymbol{b}_{n, 1}, \boldsymbol{b}_{n+1}, \boldsymbol{x}_{n, 1}, s_{n} w_{n}\right), f\left(\boldsymbol{x}_{n, 1}\right)$ is the probability density function (PDF) of the multivariate normal distribution $\boldsymbol{X}_{n, 1} \sim \mathcal{N}\left(\mathbf{1}, \mathbf{D}^{-1} \boldsymbol{\Sigma}_{n, 1} \mathbf{D}^{-1}\right)$, and $\mathbf{D}=\operatorname{diag}\left(\boldsymbol{m}_{n}\right)$ $\left(\arg \max _{\boldsymbol{b}_{n, 1} \in \Delta^{d-1}} s_{n} w_{n} \mathbb{E}\left[\left\langle\boldsymbol{b}_{n, 1}, \boldsymbol{X}_{n, 1}\right\rangle W_{n, 1}\right]\right.$ is equivalent to $\arg \max _{\boldsymbol{b}_{n, 1} \in \Delta^{d-1}} w_{n} \mathbb{E}\left[\left\langle\boldsymbol{b}_{n, 1}, \boldsymbol{X}_{n, 1}\right\rangle W_{n, 1}\right]$ as the gross wealth at the end of the $n$-th day $s_{n}=s_{0} \prod_{i=1}^{n}\left\langle\boldsymbol{b}_{i}, \boldsymbol{x}_{i}\right\rangle$ is independent of $\left.\boldsymbol{b}_{n, 1}\right)$. As a result, not only forecasting the covariance matrix of price changes between time 0 and 1 after the end of the $n$-th day $\boldsymbol{\Sigma}_{n, 1}$ (i.e. multivariate intraday volatility), but also calculating the Monte Carlo numerical integration (a closed-form solution of $\mathbb{E}\left[S_{n+1} \mid \mathcal{F}_{n}\right]$ does not exist as that of $w_{n, 1}$ does not) is required to obtain $\boldsymbol{b}_{n, 1}^{*}$.

Therefore, to avoid both the intraday forecasting of volatility and the heavy computation of integration (ultimately, to make the stochastic programming problem in Equation (21) simpler), the 


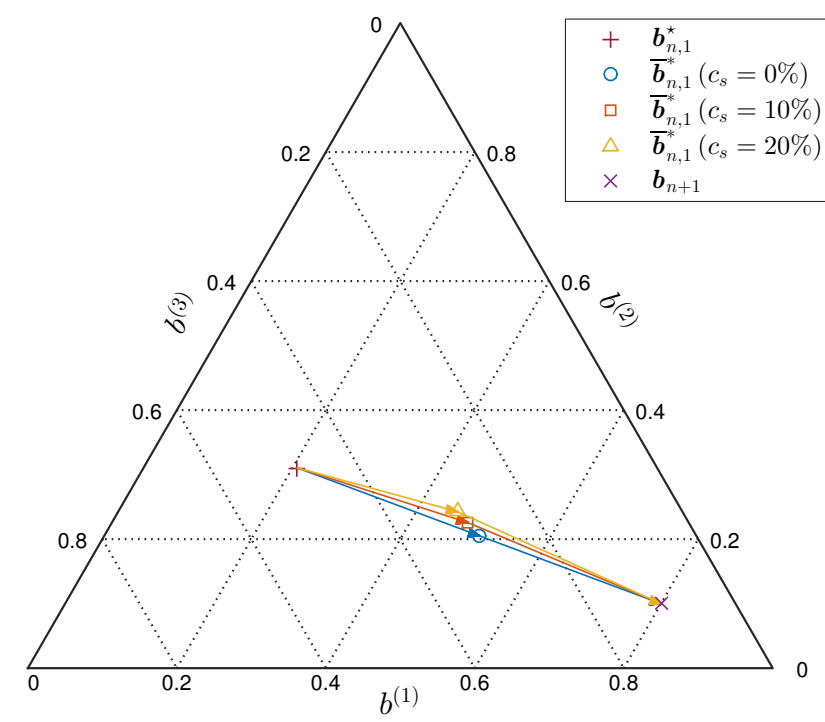

(a) $\nu_{n-1,1}$ is USD $9 \times 10^{5}$.

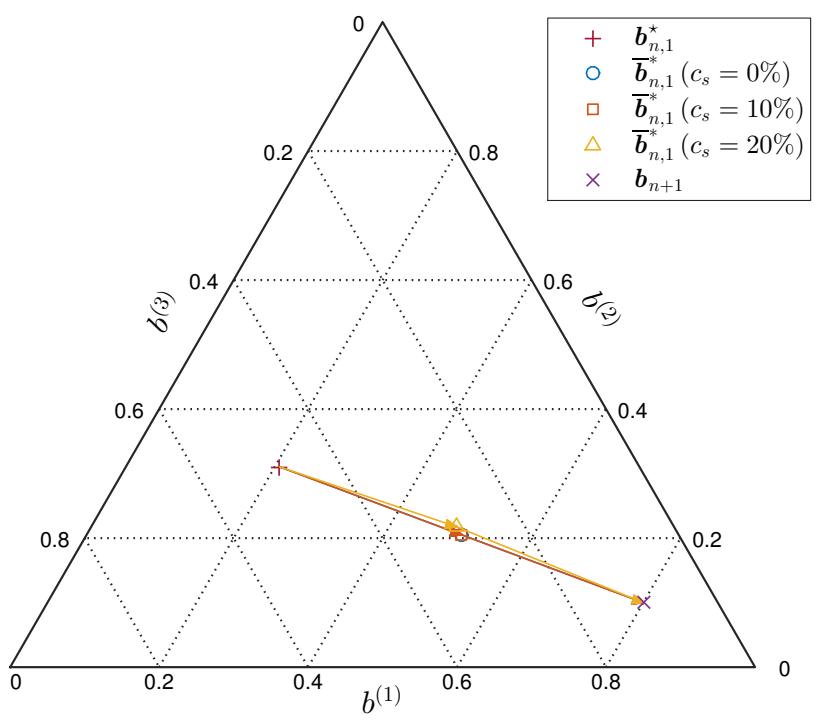

(b) $\nu_{n-1,1}$ is USD $2 \times 10^{6}$.

Figure 3. The suboptimal path of single intraday trading is dependent on $c_{p}, c_{s}$, and $\nu_{n-1,1}\left(c_{p}=0\right.$, $\boldsymbol{b}_{n}=\left[\begin{array}{lll}1 / 3 & 1 / 3 & 1 / 3\end{array}\right]^{\mathrm{T}}, \boldsymbol{b}_{n+1}=\left[\begin{array}{lll}0.8 & 0.1 & 0.1\end{array}\right]^{\mathrm{T}}$, and $\left.\boldsymbol{x}_{n, 0}=\left[\begin{array}{lll}0.6 & 0.9 & 1.4\end{array}\right]^{\mathrm{T}}\right)$. 10-level limit order book data of AAPL $\left(b^{(1)}\right)$, AMZN $\left(b^{(2)}\right)$, and GOOG $\left(b^{(3)}\right)$ on 21 Jun 2012 at 16:00:00 was used.

expected value solution of the stochastic programming (suboptimal solution of $\boldsymbol{b}_{n, 1}^{*}$ ) is calculated by replacing the random variable $\boldsymbol{X}_{n, 1}$ in Equation (21) with its expected value $\mathbb{E}\left[\boldsymbol{X}_{n, 1}\right]=\mathbf{1}$ (Birge and Louveaux 2011, p. 165):

$$
\begin{aligned}
\overline{\boldsymbol{b}}_{n, 1}^{*} & =\underset{\boldsymbol{b}_{n, 1} \in \Delta^{d-1}}{\arg \max } w_{n}\left\langle\boldsymbol{b}_{n, 1}, \mathbf{1}\right\rangle \bar{w}_{n, 1} \\
& =\underset{\boldsymbol{b}_{n, 1} \in \Delta^{d-1}}{\arg \max } w_{n} \bar{w}_{n, 1},
\end{aligned}
$$

where $\bar{w}_{n, 1}=w\left(\boldsymbol{b}_{n, 1}, \boldsymbol{b}_{n+1}, \mathbf{1}, s_{n} w_{n}\right)$ (the unimodality of $w_{n} \bar{w}_{n, 1}$ with respect to $\boldsymbol{b}_{n, 1} \in \Delta^{d-1}$ is not proved in this paper although an example is provided in Appendix A; therefore, a local optimum is not guaranteed to be a global optimum). $\overline{\boldsymbol{b}}_{n, 1}^{*}$ is always worse than or equal to $\boldsymbol{b}_{n, 1}^{*}$ in terms of the value of the stochastic solution (VSS), defined as the loss by not considering the random variations (Birge and Louveaux 2011, p. 9):

$$
w_{n}^{*} \mathbb{E}\left[\left\langle\boldsymbol{b}_{n, 1}^{*}, \boldsymbol{X}_{n, 1}\right\rangle W_{n, 1}^{*}\right]-\bar{w}_{n}^{*} \mathbb{E}\left[\left\langle\overline{\boldsymbol{b}}_{n, 1}^{*}, \boldsymbol{X}_{n, 1}\right\rangle \bar{W}_{n, 1}^{*}\right] \geq 0
$$

where

- $w_{n}^{*}=w\left(\boldsymbol{b}_{n}, \boldsymbol{b}_{n, 1}^{*}, \boldsymbol{x}_{n, 0}, \nu_{n-1,1}\right)$,

- $\bar{w}_{n}^{*}=w\left(\boldsymbol{b}_{n}, \overline{\boldsymbol{b}}_{n, 1}^{*}, \boldsymbol{x}_{n, 0}, \nu_{n-1,1}\right)$,

- $W_{n, 1}^{*}=w\left(\boldsymbol{b}_{n, 1}^{*}, \boldsymbol{b}_{n+1}, \boldsymbol{X}_{n, 1}, s_{n} w_{n}^{*}\right)$,

- and $\bar{W}_{n, 1}^{*}=w\left(\overline{\boldsymbol{b}}_{n, 1}^{*}, \boldsymbol{b}_{n+1}, \boldsymbol{X}_{n, 1}, s_{n} \bar{w}_{n}^{*}\right)$

(VSS is always non-negative for any stochastic program because $\boldsymbol{b}_{n, 1}^{*}$ is an optimal solution, while $\overline{\boldsymbol{b}}_{n, 1}^{*}$ is just one solution of $\arg \max _{\boldsymbol{b} \in \Delta^{d-1}} \mathbb{E}\left[S_{n+1} \mid \mathcal{F}_{n}\right]$ (Birge and Louveaux 2011, p. 166)). However, the suboptimal solution $\overline{\boldsymbol{b}}_{n, 1}^{*}$, obtainable by nonlinear programming, has the merits of computational simplicity by neither forecasting the intraday volatility nor conducting the Monte Carlo numerical integration. 
$\overline{\boldsymbol{b}}_{n, 1}^{*}$ is a function of $c_{p}, c_{s}$, and $\nu_{n-1,1}$ as shown in Figure 3 because $w_{n}$ and $\bar{w}_{n, 1}$ are a function of $c_{p}, c_{s}$, and $\nu_{n-1,1}$. To be specific, each arrow in Figure 3 indicates a suboptimal trading path from the starting point:

$$
\boldsymbol{b}_{n, 1}^{\star} \stackrel{\text { def }}{=} \frac{\boldsymbol{b}_{n} \odot \boldsymbol{x}_{n, 0}}{\left\langle\boldsymbol{b}_{n}, \boldsymbol{x}_{n, 0}\right\rangle}
$$

where $\odot$ denotes element-wise multiplication of vectors $\left(w\left(\boldsymbol{b}_{n}, \boldsymbol{b}_{n, 1}^{\star}, \boldsymbol{x}_{n, 0}, \nu_{n-1,1}\right)=1\right.$ is always satisfied), to the end point $\boldsymbol{b}_{n+1}$. The starting point is $\boldsymbol{b}_{n, 1}^{\star}$, not $\boldsymbol{b}_{n}\left(\boldsymbol{b}_{n, 1}^{\star}\right.$ is equivalent to $\boldsymbol{b}_{n}$ if there are no price changes). This is because $\boldsymbol{b}_{n}$ changes over time as prices change even if we do not rebalance a portfolio, as shown in Figure 9(a). Equation (24) indicates that the weight of the $j$-th asset $b_{n, 1}^{\star(j)}$ increases (decreases) as the current price of the $j$-th asset $m_{n, 0}^{(j)}$ has increased (decreased) compared to the previous price of the $j$-th asset $m_{n-1,1}^{(j)}$ (i.e. if we do not trade at time 1 after the end of the $n$-th day, then $\boldsymbol{b}_{n, 1}=\boldsymbol{b}_{n, 1}^{\star}$ is satisfied.

\subsection{Multiple intraday trades $(\tau \geq 2)$}

If more than one intraday trading per trading day has been considered, then the conditional expected value of the gross wealth at the end of the $(n+1)$-th day, given the past observations $\boldsymbol{x}_{1}, \boldsymbol{x}_{2}, \ldots, \boldsymbol{x}_{n}$, and using Equation (6), (7), and (12), is given by

$$
\mathbb{E}\left[S_{n+1} \mid \mathcal{F}_{n}\right]=s_{n} w_{n} \mathbb{E}\left[\left(\prod_{t=1}^{\tau}\left\langle\boldsymbol{b}_{n, t}, \boldsymbol{X}_{n, t}\right\rangle W_{n, t}\right)\left\langle\boldsymbol{b}_{n+1}, \boldsymbol{X}_{n+1,0}\right\rangle\right]
$$

where

- $w_{n}=w\left(\boldsymbol{b}_{n}, \boldsymbol{b}_{n, 1}, \boldsymbol{x}_{n, 0}, \nu_{n-1, \tau}\right)$,

$$
\circ \boldsymbol{x}_{n, 0}=\left[\frac{m_{n}^{(1)}}{m_{n-1, \tau}^{(1)}} \frac{m_{n}^{(2)}}{m_{n-1, \tau}^{(2)}} \cdots \frac{m_{n}^{(d)}}{m_{n-1, \tau}^{(d)}}\right]^{\mathrm{T}}
$$

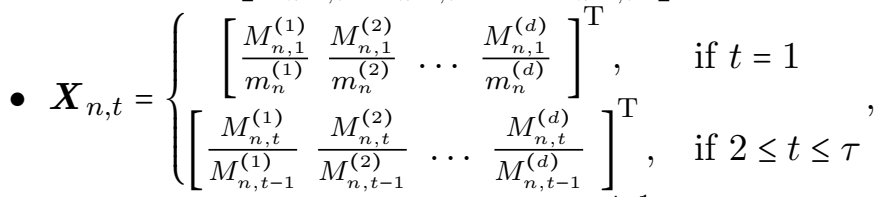

- $W_{n, t}=w\left(\boldsymbol{b}_{n, t}, \boldsymbol{b}_{n, t+1}, \boldsymbol{X}_{n, t}, s_{n} w_{n} \prod_{t^{\prime}=1}^{t-1}\left\langle\boldsymbol{b}_{n, t^{\prime}}, \boldsymbol{X}_{n, t^{\prime}}\right\rangle W_{n, t^{\prime}}\right)$,

$\circ \prod_{t^{\prime}=1}^{0}(\cdot) \stackrel{\text { def }}{=} 1$,

○ $\boldsymbol{b}_{n, \tau+1} \stackrel{\text { def }}{=} \boldsymbol{b}_{n+1}$,

- and $\boldsymbol{X}_{n+1,0}=\left[\frac{M_{n+1}^{(1)}}{M_{n, \tau}^{(1)}} \frac{M_{n+1}^{(2)}}{M_{n, \tau}^{(2)}} \cdots \frac{M_{n+1}^{(d)}}{M_{n, \tau}^{(d)}}\right]^{\mathrm{T}}$.

By Assumption 5.1 that the random vectors $\boldsymbol{X}_{n, 1}, \boldsymbol{X}_{n, 2}, \ldots, \boldsymbol{X}_{n, \tau}, \boldsymbol{X}_{n+1,0}$ are mutually independent, Equation (25) can be rewritten as

$$
\mathbb{E}\left[S_{n+1} \mid \mathcal{F}_{n}\right]=s_{n} w_{n} \mathbb{E}\left[\prod_{t=1}^{\tau}\left\langle\boldsymbol{b}_{n, t}, \boldsymbol{X}_{n, t}\right\rangle W_{n, t}\right] \mathbb{E}\left[\left\langle\boldsymbol{b}_{n+1}, \boldsymbol{X}_{n+1,0}\right\rangle\right],
$$

but $\left\langle\boldsymbol{b}_{n, t}, \boldsymbol{X}_{n, t}\right\rangle$ and $W_{n, t}$ are mutually dependent because $W_{n, t}$ is a function of $\boldsymbol{X}_{n, t}$. Also, $\left\langle\boldsymbol{b}_{n, t}, \boldsymbol{X}_{n, t}\right\rangle W_{n, t}$ and $\left\langle\boldsymbol{b}_{n, t^{\prime}}, \boldsymbol{X}_{n, t^{\prime}}\right\rangle W_{n, t^{\prime}}$ are mutually dependent, where $t \neq t^{\prime}$, because $W_{n, t}$ is a function of $\boldsymbol{X}_{n, 1}, \boldsymbol{X}_{n, 2}, \ldots, \boldsymbol{X}_{n, t-1}$ as well as $\boldsymbol{X}_{n, t}$. Finally, by using the property of $\mathbb{E}\left[\left\langle\boldsymbol{b}_{n+1}, \boldsymbol{X}_{n+1,0}\right\rangle\right]=1$, 
as proved in Equation (17), $\mathbb{E}\left[S_{n+1} \mid \mathcal{F}_{n}\right]$ in Equation (25) can be simplified as

$$
\mathbb{E}\left[S_{n+1} \mid \mathcal{F}_{n}\right]=s_{n} w_{n} \mathbb{E}\left[\prod_{t=1}^{\tau}\left\langle\boldsymbol{b}_{n, t}, \boldsymbol{X}_{n, t}\right\rangle W_{n, t}\right]
$$

Our goal is to find the optimal portfolio vectors $\boldsymbol{b}_{n, 1}^{*}, \boldsymbol{b}_{n, 2}^{*}, \ldots, \boldsymbol{b}_{n, \tau}^{*}$ that maximise $\mathbb{E}\left[S_{n+1} \mid \mathcal{F}_{n}\right]$, and this is a stochastic programming problem as follows:

$$
\begin{aligned}
& \boldsymbol{b}_{n, 1}^{*}, \boldsymbol{b}_{n, 2}^{*}, \ldots, \boldsymbol{b}_{n, \tau}^{\star} \\
= & \underset{\boldsymbol{b}_{n, 1}, \boldsymbol{b}_{n, 2}, \ldots, \boldsymbol{b}_{n, \tau} \in \Delta^{d-1}}{\arg \max } \mathbb{E}\left[S_{n+1} \mid \mathcal{F}_{n}\right] \\
= & \underset{\boldsymbol{b}_{n, 1}, \boldsymbol{b}_{n, 2}, \ldots, \boldsymbol{b}_{n, \tau} \in \Delta^{d-1}}{\arg \max } s_{n} w_{n} \mathbb{E}\left[\prod_{t=1}^{\tau}\left\langle\boldsymbol{b}_{n, t}, \boldsymbol{X}_{n, t}\right\rangle W_{n, t}\right] \\
= & \underset{\boldsymbol{b}_{n, 1}, \boldsymbol{b}_{n, 2}, \ldots, \boldsymbol{b}_{n, \tau} \in \Delta^{d-1}}{\arg \max } w_{n} \mathbb{E}\left[\prod_{t=1}^{\tau}\left\langle\boldsymbol{b}_{n, t}, \boldsymbol{X}_{n, t}\right\rangle W_{n, t}\right] \\
= & \underset{\boldsymbol{b}_{n, 1}, \boldsymbol{b}_{n, 2}, \ldots, \boldsymbol{b}_{n, \tau} \in \Delta^{d-1}}{\arg \max } w_{n} \int_{\boldsymbol{x}_{n, 1} \in \mathbb{R}_{+}^{d}} \int_{\boldsymbol{x}_{n, 2} \in \mathbb{R}_{+}^{d}} \cdots \int_{\boldsymbol{x}_{n, \tau} \in \mathbb{R}_{+}^{d}} \prod_{t=1}^{\tau}\left\langle\boldsymbol{b}_{n, t}, \boldsymbol{x}_{n, t}\right\rangle w_{n, t} f\left(\boldsymbol{x}_{n, t}\right) d \boldsymbol{x}_{n, 1} d \boldsymbol{x}_{n, 2} \cdots d \boldsymbol{x}_{n, \tau},
\end{aligned}
$$

where

- $w_{n, t}=w\left(\boldsymbol{b}_{n, t}, \boldsymbol{b}_{n, t+1}, \boldsymbol{x}_{n, t}, s_{n} w_{n} \prod_{t^{\prime}=1}^{t-1}\left\langle\boldsymbol{b}_{n, t^{\prime}}, \boldsymbol{x}_{n, t^{\prime}}\right\rangle w_{n, t^{\prime}}\right)$,

$$
\text { ○ } \boldsymbol{b}_{n, \tau+1} \stackrel{\text { def }}{=} \boldsymbol{b}_{n+1} \text {, }
$$

- $f\left(\boldsymbol{x}_{n, t}\right)$ is the PDF of the multivariate normal distribution $\mathcal{N}\left(\mathbf{1}, \mathbf{D}_{n, t-1}^{-1} \boldsymbol{\Sigma}_{n, t} \mathbf{D}_{n, t-1}^{-1}\right)$,

$$
\circ \text { and } \mathbf{D}_{n, t-1}=\left\{\begin{array}{cl}
\operatorname{diag}\left(\boldsymbol{m}_{n}\right), & \text { if } t=1 \\
\operatorname{diag}\left(\boldsymbol{M}_{n, t-1}\right), & \text { if } 2 \leq t \leq \tau
\end{array}\right.
$$

and $\arg \max _{\boldsymbol{b}_{n, 1}, \boldsymbol{b}_{n, 2}, \ldots, \boldsymbol{b}_{n, \tau} \in \Delta^{d-1}} s_{n} w_{n} \mathbb{E}\left[\prod_{t=1}^{\tau}\left\langle\boldsymbol{b}_{n, t}, \boldsymbol{X}_{n, t}\right\rangle W_{n, t}\right]$

is equivalent to $\arg \max _{\boldsymbol{b}_{n, 1}, \boldsymbol{b}_{n, 2}, \ldots, \boldsymbol{b}_{n, \tau} \in \Delta^{d-1}} w_{n} \mathbb{E}\left[\prod_{t=1}^{\tau}\left\langle\boldsymbol{b}_{n, t}, \boldsymbol{X}_{n, t}\right\rangle W_{n, t}\right]$ since the gross wealth at the end of the $n$-th day $s_{n}=s_{0} \prod_{i=1}^{n}\left\langle\boldsymbol{b}_{i}, \boldsymbol{x}_{i}\right\rangle$ is independent of $\boldsymbol{b}_{n, 1}, \boldsymbol{b}_{n, 2}, \ldots, \boldsymbol{b}_{n, \tau}$. Similar to the one intraday trading case, here not only forecasting the covariance matrix of price changes between time $t-1$ and $t$ after the end of the $n$-th day $\boldsymbol{\Sigma}_{n, t}$, but also calculating the Monte Carlo numerical integration is required to obtain $\boldsymbol{b}_{n, 1}^{*}, \boldsymbol{b}_{n, 2}^{*}, \ldots, \boldsymbol{b}_{n, \tau}^{*}$.

Once again, to make the stochastic programming problem in Equation (28) simpler, the expected value solution of the stochastic programming (a suboptimal solution of $\boldsymbol{b}_{n, 1}^{*}, \boldsymbol{b}_{n, 2}^{*}, \ldots, \boldsymbol{b}_{n, \tau}^{*}$ ) is calculated by replacing all the random variables $\boldsymbol{X}_{n, 1}, \boldsymbol{X}_{n, 2}, \ldots, \boldsymbol{X}_{n, \tau}$ in Equation (28) with their expected values $\mathbb{E}\left[\boldsymbol{X}_{n, 1}\right]=\mathbb{E}\left[\boldsymbol{X}_{n, 2}\right]=\ldots=\mathbb{E}\left[\boldsymbol{X}_{n, \tau}\right]=\mathbf{1}$ (Birge and Louveaux 2011, p. 165):

$$
\begin{aligned}
\overline{\boldsymbol{b}}_{n, 1}^{*}, \overline{\boldsymbol{b}}_{n, 2}^{*}, \cdots, \overline{\boldsymbol{b}}_{n, \tau}^{*} & =\underset{\boldsymbol{b}_{n, 1}, \boldsymbol{b}_{n, 2}, \ldots, \boldsymbol{b}_{n, \tau} \in \Delta^{d-1}}{\arg \max } w_{n} \prod_{t=1}^{\tau}\left\langle\boldsymbol{b}_{n, t}, \boldsymbol{1}\right\rangle \bar{w}_{n, t} \\
& =\underset{\boldsymbol{b}_{n, 1}, \boldsymbol{b}_{n, 2}, \ldots, \boldsymbol{b}_{n, \tau} \in \Delta^{d-1}}{\arg \max } w_{n} \prod_{t=1}^{\tau} \bar{w}_{n, t},
\end{aligned}
$$

where $\bar{w}_{n, t}=w\left(\boldsymbol{b}_{n, t}, \boldsymbol{b}_{n, t+1}, \mathbf{1}, s_{n} w_{n} \prod_{t^{\prime}=1}^{t-1} \bar{w}_{n, t^{\prime}}\right)$, and $\boldsymbol{b}_{n, \tau+1} \stackrel{\text { def }}{=} \boldsymbol{b}_{n+1}$ (the unimodality of $w_{n} \prod_{t=1}^{\tau} \bar{w}_{n, t}$ with respect to $\boldsymbol{b}_{n, 1}, \boldsymbol{b}_{n, 2}, \ldots, \boldsymbol{b}_{n, \tau} \in \Delta^{d-1}$ is not proved in this paper; therefore, a local optimum is not guaranteed to be a global optimum). $\overline{\boldsymbol{b}}_{n, 1}^{*}, \overline{\boldsymbol{b}}_{n, 2}^{*}, \ldots, \overline{\boldsymbol{b}}_{n, \tau}^{*}$ is always worse than or equal to $\boldsymbol{b}_{n, 1}^{*}, \boldsymbol{b}_{n, 2}^{*}, \ldots, \boldsymbol{b}_{n, \tau}^{*}$ in terms of the value of the stochastic solution (VSS), defined as the loss by not 


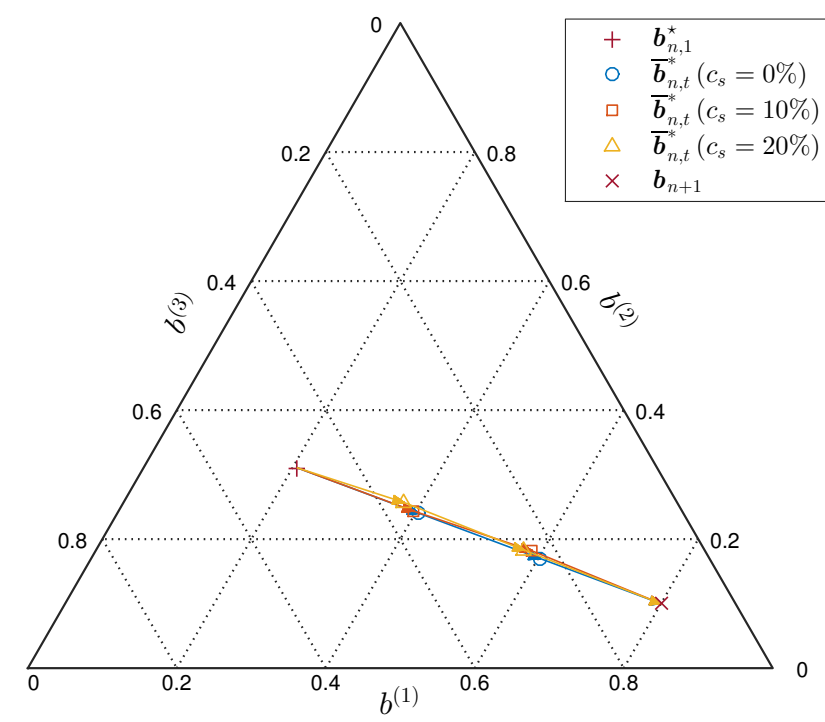

(a) $\nu_{n-1, \tau}$ is USD $9 \times 10^{5}$.

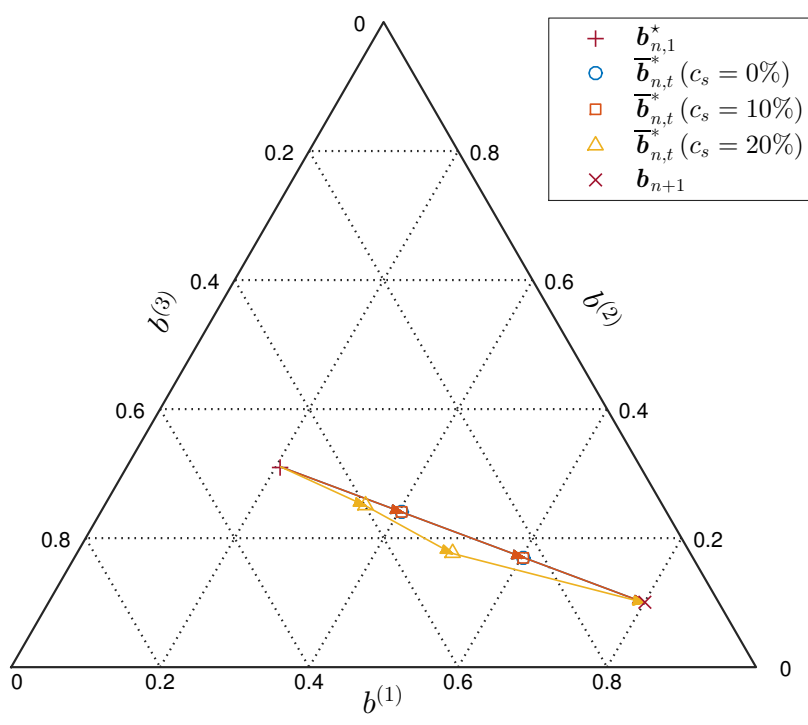

(b) $\nu_{n-1, \tau}$ is USD $3 \times 10^{6}$.

Figure 4. The suboptimal path of two intraday trades is dependent on $c_{p}, c_{s}$, and $\nu_{n-1, \tau}\left(c_{p}=0, \boldsymbol{b}_{n}=[1 / 31 / 31 / 3]^{\mathrm{T}}\right.$, $\boldsymbol{b}_{n+1}=\left[\begin{array}{lll}0.8 & 0.1 & 0.1\end{array}\right]^{\mathrm{T}}$, and $\left.\boldsymbol{x}_{n, 0}=\left[\begin{array}{lll}0.6 & 0.9 & 1.4\end{array}\right]^{\mathrm{T}}\right)$. 10-level limit order book data of AAPL $\left(b^{(1)}\right)$, AMZN $\left(b^{(2)}\right)$, and GOOG $\left(b^{(3)}\right)$ on 21 Jun 2012 at 16:00:00 was used.

considering the random variations (Birge and Louveaux 2011, p. 9):

$$
w_{n}^{*} \mathbb{E}\left[\prod_{t=1}^{\tau}\left\langle\boldsymbol{b}_{n, t}^{*}, \boldsymbol{X}_{n, t}\right\rangle W_{n, t}^{*}\right]-\bar{w}_{n}^{*} \mathbb{E}\left[\prod_{t=1}^{\tau}\left\langle\overline{\boldsymbol{b}}_{n, t}^{*}, \boldsymbol{X}_{n, t}\right\rangle \bar{W}_{n, t}^{*}\right] \geq 0
$$

where

- $w_{n}^{*}=w\left(\boldsymbol{b}_{n}, \boldsymbol{b}_{n, 1}^{*}, \boldsymbol{x}_{n, 0}, \nu_{n-1, \tau}\right)$,

- $\bar{w}_{n}^{*}=w\left(\boldsymbol{b}_{n}, \overline{\boldsymbol{b}}_{n, 1}^{*}, \boldsymbol{x}_{n, 0}, \nu_{n-1, \tau}\right)$,

- $W_{n, t}^{*}=w\left(\boldsymbol{b}_{n, t}^{*}, \boldsymbol{b}_{n, t+1}^{*}, \boldsymbol{X}_{n, t}, s_{n} w_{n}^{*} \prod_{t^{\prime}=1}^{t-1}\left\langle\boldsymbol{b}_{n, t^{\prime}}^{*}, \boldsymbol{X}_{n, t^{\prime}}\right\rangle W_{n, t^{\prime}}^{*}\right)$,

○ $\boldsymbol{b}_{n, \tau+1}^{*} \stackrel{\text { def }}{=} \boldsymbol{b}_{n+1}$,

- $\bar{W}_{n, t}^{*}=w\left(\overline{\boldsymbol{b}}_{n, t}^{*}, \overline{\boldsymbol{b}}_{n, t+1}^{*}, \boldsymbol{X}_{n, t}, s_{n} \bar{w}_{n}^{*} \prod_{t^{\prime}=1}^{t-1}\left\langle\overline{\boldsymbol{b}}_{n, t^{\prime}}^{*}, \boldsymbol{X}_{n, t^{\prime}}\right\rangle \bar{W}_{n, t^{\prime}}^{*}\right)$,

$\circ$ and $\overline{\boldsymbol{b}}_{n, \tau+1}^{*} \stackrel{\text { def }}{=} \boldsymbol{b}_{n+1}$.

Meanwhile, forecasting intraday returns can be performed to find a more optimal solution than $\overline{\boldsymbol{b}}_{n, 1}^{*}, \overline{\boldsymbol{b}}_{n, 2}^{*}, \ldots, \overline{\boldsymbol{b}}_{n, \tau}^{*}$. Firstly, if forecasting intraday expected returns is possible, Equation (29) can be rewritten as the following equation by replacing 1 in Equation (29) with $\mathbb{E}\left[\boldsymbol{X}_{n, t}\right]$ :

$$
\overline{\boldsymbol{b}}_{n, 1}^{*}, \overline{\boldsymbol{b}}_{n, 2}^{*}, \cdots, \overline{\boldsymbol{b}}_{n, \tau}^{*}=\underset{\boldsymbol{b}_{n, 1}, \boldsymbol{b}_{n, 2}, \ldots, \boldsymbol{b}_{n, \tau} \in \Delta^{d-1}}{\arg \max } w_{n} \prod_{t=1}^{\tau}\left\langle\boldsymbol{b}_{n, t}, \mathbb{E}\left[\boldsymbol{X}_{n, t}\right]\right\rangle \bar{w}_{n, t},
$$

where $\bar{w}_{n, t}=w\left(\boldsymbol{b}_{n, t}, \boldsymbol{b}_{n, t+1}, \mathbb{E}\left[\boldsymbol{X}_{n, t}\right], s_{n} w_{n} \prod_{t^{\prime}=1}^{t-1}\left\langle\boldsymbol{b}_{n, t^{\prime}}, \mathbb{E}\left[\boldsymbol{X}_{n, t^{\prime}}\right]\right\rangle \bar{w}_{n, t^{\prime}}\right)$, and $\boldsymbol{b}_{n, \tau+1} \stackrel{\text { def }}{=} \boldsymbol{b}_{n+1}$. The computational burden of Equation (31) is the same as that of Equation (29) because the numerical integration is not performed in both the cases. Secondly, intraday (each day is divided into 10-minute intervals) volatility forecasting can be employed by using the univariate GARCH model ( $\boldsymbol{\Sigma}_{n, t}$ is assumed as a diagonal matrix) by Engle and Sokalska (2012). However, calculating integrations in Equation (28) is required, which in turn causes a heavy computational burden. Lastly, the optimal solution $\boldsymbol{b}_{n, 1}^{*}, \boldsymbol{b}_{n, 2}^{*}, \ldots, \boldsymbol{b}_{n, \tau}^{*}$ in Equation (28) can be obtained by forecasting both intraday expected returns and volatilities. 
$\overline{\boldsymbol{b}}_{n, 1}^{*}, \overline{\boldsymbol{b}}_{n, 2}^{*}, \ldots, \overline{\boldsymbol{b}}_{n, \tau}^{*}$ is a function of $c_{p}, c_{s}$, and $\nu_{n-1, \tau}$ as shown in Figure 4 because $w_{n}$ and $\bar{w}_{n, t}, \forall t \in\{1,2, \ldots, \tau\}$ are functions of $c_{p}, c_{s}$, and $\nu_{n-1, \tau}$. To be specific, each arrow indicates a suboptimal trading path from the starting point $\boldsymbol{b}_{n, 1}^{\star}=\frac{\boldsymbol{b}_{n} \odot \boldsymbol{x}_{n, 0}}{\left\langle\boldsymbol{b}_{n}, \boldsymbol{x}_{n, 0}\right\rangle}$ in Equation (24) to the end point $\boldsymbol{b}_{n+1}$.

A recommended initial value of $\boldsymbol{b}_{n, t}$ for the optimisation of Equation (29) is

$$
\boldsymbol{b}_{n, t}^{\dagger}=\boldsymbol{b}_{n, 1}^{\star}+\frac{t}{\tau+1}\left(\boldsymbol{b}_{n+1}-\boldsymbol{b}_{n, 1}^{\star}\right)
$$

where $t \in\{1,2, \ldots, \tau\}$ (i.e. $\boldsymbol{b}_{n, t}^{\dagger}, \forall t$ is linearly located between $\boldsymbol{b}_{n, 1}^{\star}$ and $\boldsymbol{b}_{n+1}$ with the same distance). This is because the suboptimal portfolio vector $\overline{\boldsymbol{b}}_{n, t}^{*}, \forall t$ is not far from $\boldsymbol{b}_{n, t}^{\dagger}, \forall t$ as shown in Figure 3 and 4 . As a result, using the initial value $\boldsymbol{b}_{n, t}^{\dagger}$ will reduce computation time searching for the solution $\overline{\boldsymbol{b}}_{n, 1}^{*}, \overline{\boldsymbol{b}}_{n, 2}^{*}, \cdots, \overline{\boldsymbol{b}}_{n, \tau}^{*}$ in Equation (29).

\subsection{Optimal number of intraday trades}

The optimal number of intraday trades $\tau^{*}$ can be written as

$$
\tau^{*}=\underset{\tau \in\{0,1, \ldots\}}{\arg \max } \bar{s}_{n+1}(\tau),
$$

where $\bar{s}_{n+1}(\tau)$ is the gross wealth at the end of the $(n+1)$-th day i) when a portfolio is rebalanced through the suboptimal trading path $\overline{\boldsymbol{b}}_{n, 1}^{*}, \overline{\boldsymbol{b}}_{n, 2}^{*}, \ldots, \overline{\boldsymbol{b}}_{n, \tau}^{*}$, given the number of intraday trades $\tau$, and ii) when LOBs do not change between time 0 and time $\tau$ after the end of the $n$-th day (this corresponds to Assumption 5.2). $\bar{s}_{n+1}(\tau)$ can be written, using Equation (17) and (29), as

$$
\bar{s}_{n+1}(\tau)=\left\{\begin{array}{cc}
s_{n} w\left(\boldsymbol{b}_{n}, \boldsymbol{b}_{n+1}, \boldsymbol{x}_{n, 0}, \nu_{n-1, \tau}\right), & \text { if } \tau=0 \\
s_{n} \bar{w}_{n}^{*} \prod_{t=1}^{\tau} \bar{w}_{n, t}^{*}, & \text { if } \tau \geq 1
\end{array},\right.
$$

where

- $\bar{w}_{n}^{*}=w\left(\boldsymbol{b}_{n}, \overline{\boldsymbol{b}}_{n, 1}^{*}, \boldsymbol{x}_{n, 0}, \nu_{n-1, \tau}\right)$,

- $\bar{w}_{n, t}^{*}=w\left(\overline{\boldsymbol{b}}_{n, t}^{*}, \overline{\boldsymbol{b}}_{n, t+1}^{*}, \mathbf{1}, s_{n} \bar{w}_{n}^{*} \prod_{t^{\prime}=1}^{t-1} \bar{w}_{n, t^{\prime}}^{*}\right)$,

$\circ$ and $\overline{\boldsymbol{b}}_{n, \tau+1}^{*} \stackrel{\text { def }}{=} \boldsymbol{b}_{n+1}$.

Also, $\bar{s}_{n+1}(\tau)$ can be rewritten, using Equation (5) and (6), as

$$
\bar{s}_{n+1}(\tau)=s_{n}-\sum_{t=0}^{\tau} \gamma_{n, t}(\tau),{ }^{3}
$$

3

$$
\begin{aligned}
\bar{s}_{n+1}(\tau) & =\nu_{n, \tau}\left\langle\boldsymbol{b}_{n+1}, \boldsymbol{1}\right\rangle \\
& =\nu_{n, \tau} \\
& =s_{n, \tau}-\gamma_{n, \tau} \\
& =\nu_{n, \tau-1}\left\langle\boldsymbol{b}_{n, \tau}, \boldsymbol{1}\right\rangle-\gamma_{n, \tau} \\
& =\nu_{n, \tau-1}-\gamma_{n, \tau} \\
& =s_{n, \tau-1}-\gamma_{n, \tau-1}-\gamma_{n, \tau} \\
& =s_{n}-\sum_{t=0}^{\tau} \gamma_{n, t} .
\end{aligned}
$$


where $\gamma_{n, t}(\tau)$ is transaction cost (TC) at time $t$ after the end of the $n$-th day, given the number of intraday trades $\tau$. By using Equation (10) and the no price change of Assumption 5.2 (i.e. $\left.m_{n, t}^{(j)}=m_{n}^{(j)}, \forall t \in\{1,2, \ldots, \tau\}\right)$, Equation (33) can be further simplified as:

$$
\begin{aligned}
& \tau^{*} \\
= & \underset{\tau \in\{0,1, \ldots\}}{\arg \min } \sum_{t=0}^{\tau} \gamma_{n, t}(\tau) \\
= & \underset{\tau \in\{0,1, \ldots\}}{\arg \min } \sum_{t=0}^{\tau}\left[\sum_{j=1}^{d}\left(\left(1-c_{s}\right) \bar{p}\left(\bar{q}_{n, t}^{(j)^{*}}(\tau)\right)-m_{n}^{(j)}\right) \bar{q}_{n, t}^{(j)^{*}}(\tau)+\left(c_{p}+c_{s}\right) \sum_{j=1}^{d}\left(\bar{p}\left(\bar{q}_{n, t}^{(j)^{*}}(\tau)\right) \bar{q}_{n, t}^{(j)^{*}}(\tau)\right)^{+}\right],
\end{aligned}
$$

where $\bar{q}_{n, t}^{(j)^{*}}(\tau)$ is the order size of asset $j$ when rebalancing a portfolio by following the suboptimal trading path from $\overline{\boldsymbol{b}}_{n, t}^{*}$ to $\overline{\boldsymbol{b}}_{n, t+1}^{*}$, given the number of intraday trades $\tau$.

Equation (36) implies that the overall TCs $\sum_{t=0}^{\tau} \gamma_{n, t}(\tau)$, consisting of proportional TCs and MICs, can be minimised if $\tau$ is large enough to make trading order size $\left|\bar{q}_{n, t}^{(j)^{*}}(\tau)\right|$ small for all $j$ and all $t \in\{0,1, \ldots, \tau\}$. Also, the small trading order is equivalent to that where all assets are traded at the best ask price $p_{1}^{(j)}$ or the best bid price $p_{-1}^{(j)}$ for all $t \in\{0,1, \ldots, \tau\}$ : $\bar{p}\left(\bar{q}_{n, t}^{(j)^{*}}\left(\tau^{*}\right)\right)= \begin{cases}p_{1}^{(j)}, & \text { if } \bar{q}_{n, t}^{(j)^{*}}\left(\tau^{*}\right)>0 \\ p_{-1}^{(j)}, & \text { if } \bar{q}_{n, t}^{(j)^{*}}\left(\tau^{*}\right)<0, \text { as } v_{-1}^{(j)} \leq \bar{q}_{n, t}^{(j)^{*}}\left(\tau^{*}\right) \leq v_{1}^{(j)}, \forall t . \text { Consequently, the overall TCs } \\ m_{n}^{(j)}, & \text { otherwise }\end{cases}$ can be minimised when $\tau=\tau^{*}$ as

$$
\begin{aligned}
& \sum_{t=0}^{\tau^{*}} \gamma_{n, t}\left(\tau^{*}\right) \\
= & \sum_{t=0}^{\tau^{*}}\left[\sum_{j=1}^{d}\left(\left(1-c_{s}\right) \bar{p}\left(\bar{q}_{n, t}^{(j)^{*}}\left(\tau^{*}\right)\right)-m_{n}^{(j)}\right) \bar{q}_{n, t}^{(j)^{*}}\left(\tau^{*}\right)+\left(c_{p}+c_{s}\right) \sum_{j=1}^{d}\left(\bar{p}\left(\bar{q}_{n, t}^{(j)^{*}}\left(\tau^{*}\right)\right) \bar{q}_{n, t}^{(j)^{*}}\left(\tau^{*}\right)\right)^{+}\right] \\
= & \sum_{t=0}^{\tau^{*}} \sum_{j=1}^{d}\left[\left(\left(1-c_{s}\right) p_{1}^{(j)}-m_{n}^{(j)}\right)\left(\bar{q}_{n, t}^{(j)^{*}}\left(\tau^{*}\right)\right)^{+}+\left(m_{n}^{(j)}-\left(1-c_{s}\right) p_{-1}^{(j)}\right)\left(-\bar{q}_{n, t}^{(j)^{*}}\left(\tau^{*}\right)\right)^{+}\right] \\
& +\left(c_{p}+c_{s}\right) \sum_{t=0}^{\tau^{*}} \sum_{j=1}^{d} p_{1}^{(j)}\left(\bar{q}_{n, t}^{(j)^{*}}\left(\tau^{*}\right)\right)^{+} \\
= & \sum_{t=0}^{\tau^{*}} \sum_{j=1}^{d}\left[\left(\left(1+c_{p}\right) p_{1}^{(j)}-m_{n}^{(j)}\right)\left(\bar{q}_{n, t}^{(j)}\left(\tau^{*}\right)\right)^{+}+\left(m_{n}^{(j)}-\left(1-c_{s}\right) p_{-1}^{(j)}\right)\left(-\bar{q}_{n, t}^{(j)^{*}}\left(\tau^{*}\right)\right)^{+}\right] \\
= & \sum_{j=1}^{d}\left[\left(\left(1+c_{p}\right) p_{1}^{(j)}-m_{n}^{(j)}\right) \sum_{t=0}^{\tau^{*}}\left(\bar{q}_{n, t}^{(j)^{*}}\left(\tau^{*}\right)\right)^{+}+\left(m_{n}^{(j)}-\left(1-c_{s}\right) p_{-1}^{(j)}\right) \sum_{t=0}^{\tau^{*}}\left(-\bar{q}_{n, t}^{(j)^{*}}\left(\tau^{*}\right)\right)^{+}\right]
\end{aligned}
$$

The optimal number of intraday trades $\tau^{*}$ in Equation (36) is not a unique number - it can be several numbers as $\tau^{*} \in\left\{\tau \in \mathbb{Z} \mid \tau \geq \tau_{\text {min }}^{*}\right\}$, where $\tau_{\text {min }}^{*}$ is the minimum optimal number of intraday trades. This is because both $\sum_{t=0}^{\tau}\left(\bar{q}_{n, t}^{(j)^{*}}(\tau)\right)^{+}$and $\sum_{t=0}^{\tau}\left(-\bar{q}_{n, t}^{(j)^{*}}(\tau)\right)^{+}$in Equation (37) are constants if $\tau \geq \tau_{\min }^{*}$. As a result, $\frac{\bar{s}_{n+1}(\tau)}{s_{n}}$ is a monotonically increasing function of $\tau$ as shown in Figure 5 , and 


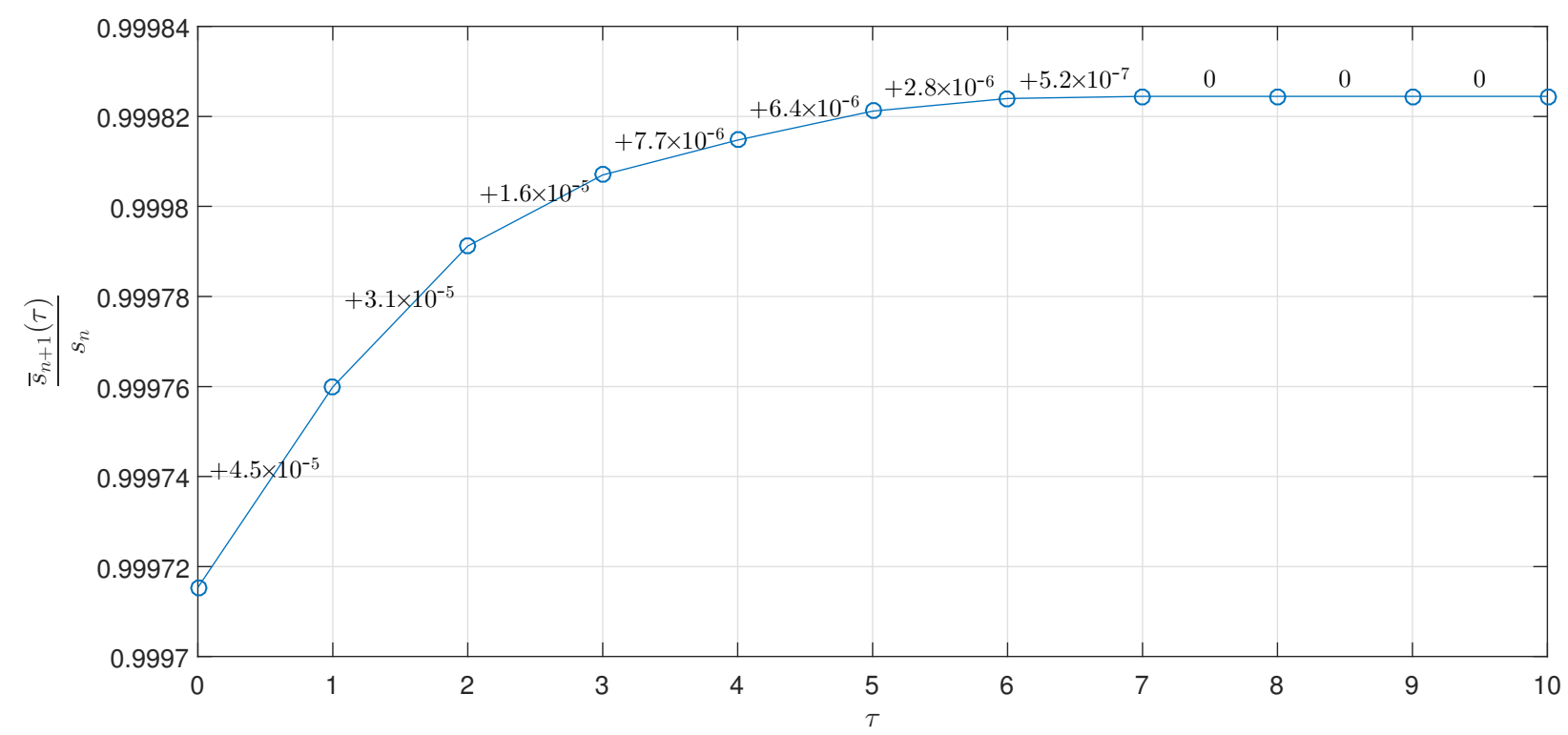

Figure 5. The optimal number of intraday trades $\tau^{*}$ is not unique $\left(c_{p}=0, c_{s}=0.00218 \%, \boldsymbol{b}_{n}=[1 / 31 / 31 / 3]^{\mathrm{T}}, \boldsymbol{b}_{n+1}=\right.$ $\left[\begin{array}{lll}0.8 & 0.1 & 0.1\end{array}\right]^{\mathrm{T}}, \boldsymbol{x}_{n, 0}=\left[\begin{array}{lll}0.6 & 0.9 & 1.4\end{array}\right]^{\mathrm{T}}$, and $\nu_{n-1, \tau}=2 \times 10^{6}$ USD). 10-level limit order book data of AAPL $\left(b^{(1)}\right)$, AMZN $\left(b^{(2)}\right)$, and GOOG $\left(b^{(3)}\right)$ on 21 Jun 2012 at 16:00:00 was used (each value above the line is the change amount of $\left.\frac{\bar{s}_{n+1}(\tau)}{s_{n}}\right)$.

$\frac{\bar{s}_{n+1}(\tau)}{s_{n}}$ does not change after $\tau=\tau_{\min }^{*}\left(\tau_{\min }^{*}\right.$ is 7 in the case of Figure 5$)$.

Algorithm 1 describes how to obtain the minimum optimal number of intraday trades $\tau_{\min }^{*}$ from the property of $v_{-1}^{(j)} \leq \bar{q}_{n, t}^{(j)^{*}}\left(\tau^{*}\right) \leq v_{1}^{(j)}, \forall j \in\{1,2, \ldots, d\}, \forall t \in\{0,1, \ldots, \tau\}$. This algorithm increases the number of intraday trades $\tau$ from 0 until either i) when $\tau$ equals the upper limit $\tau_{\max }$ (see the 5th line of Algorithm 1), where $\tau_{\max }$ is a user parameter decided by trading hours and an intraday trading interval, or ii) when trading all assets at the best ask or best bid price is possible (see the 7th line of Algorithm 1).

\subsection{Considering real-time limit order book data}

The proposed method described in Algorithm 2 is an implementation shortfall algorithm (an intraday trading strategy is determined by real-time LOB data at every intraday trading time $t$ ) for a multi-asset portfolio. It performs intraday trading by sending market orders in the following order.

(i) The portfolio vector of next day $\boldsymbol{b}_{n+1}$ is obtained from an OPS algorithm (see the 2nd line of Algorithm 2) at the end of every trading day (the end of trading day is the market opening, not midnight; see Figure 2).

(ii) The current LOBs of all assets in the portfolio are taken into account at every intraday trading time $t$ until either the time that satisfies $t=\tau_{\max }$ (see the 3rd line of Algorithm 2) or the time that satisfies $\left(v_{-1}^{(j)}\right)_{n, t} \leq q_{n, t}^{(j)} \leq\left(v_{1}^{(j)}\right)_{n, t}, \forall j$ (i.e. whether trading all assets in the current best ask or best bid price is possible or not is checked on every $t$; see the 10th line of Algorithm 2), whichever happens first.

(iii) If any of the inequalities at the 10th line of Algorithm 2 are false, then the minimum optimal number of intraday trades $\tau_{\min }^{*}$ is obtained by considering the current LOBs and by using Algorithm 1 (see between the 15th line and the 21st line of Algorithm 2).

(iv) Among the suboptimal path components $\overline{\boldsymbol{b}}_{n, t+1}^{*}, \overline{\boldsymbol{b}}_{n, t+2}^{*}, \ldots, \overline{\boldsymbol{b}}_{n, \tau}^{*}$, calculated at the $16 \mathrm{th}$ line of Algorithm 2, only one component $\overline{\boldsymbol{b}}_{n, t+1}^{*}$ is used for the rebalancing at time $t$ (see the 22nd line of Algorithm 2), but the other components of the suboptimal path $\overline{\boldsymbol{b}}_{n, t+2}^{*}, \overline{\boldsymbol{b}}_{n, t+3}^{*}, \ldots, \overline{\boldsymbol{b}}_{n, \tau}^{*}$ are ignored. This is because new LOBs will be given at time $t+1$ (see the 8th line of 


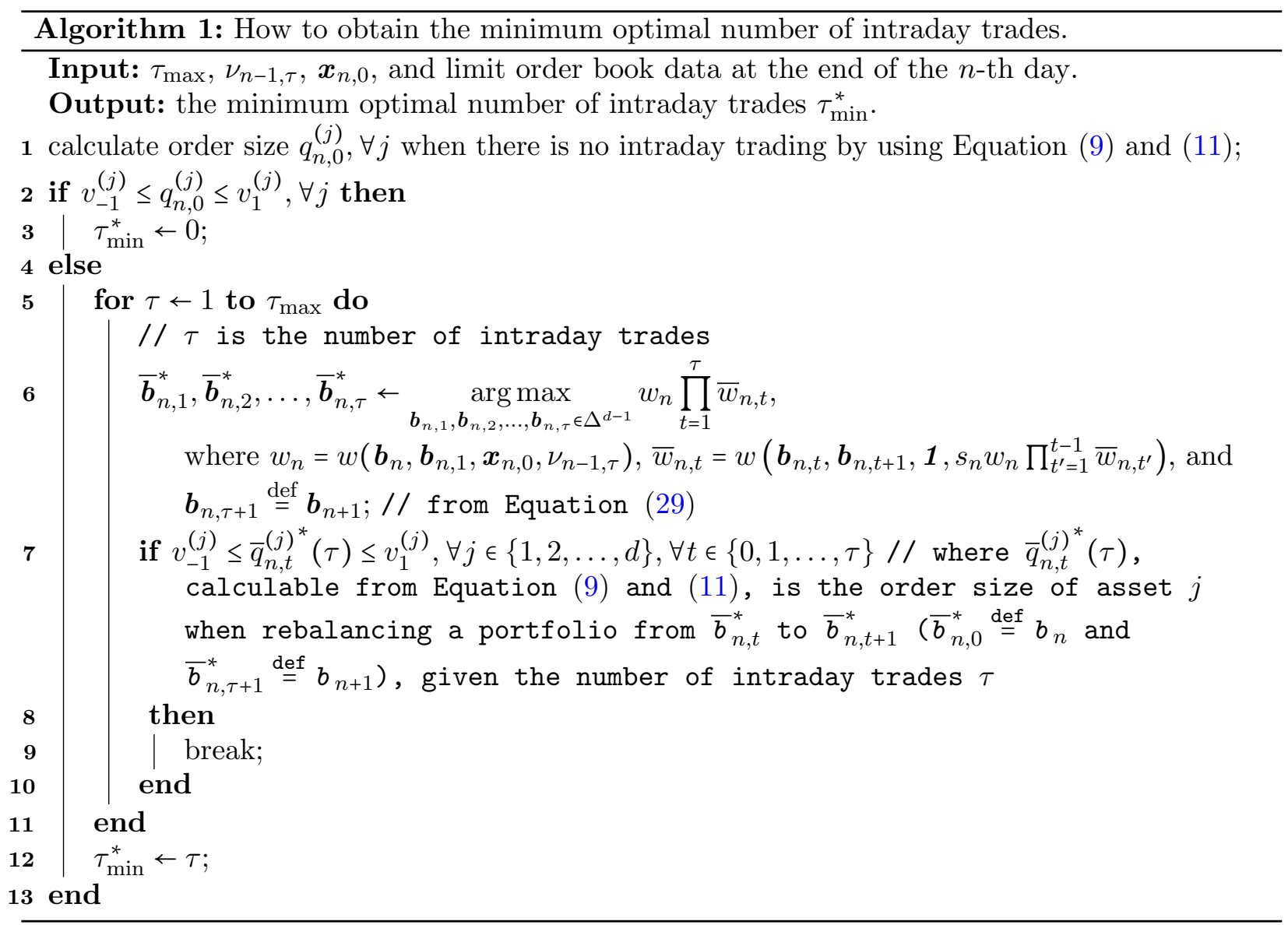

Algorithm 2).

However, Algorithm 2 ignores the risk of intraday price volatility as Alfonsi et al. $(2008,2010)$ did (i.e. the volatility of the random intraday market vectors $\boldsymbol{X}_{n, t+1}, \boldsymbol{X}_{n, t+2}, \ldots, \boldsymbol{X}_{n, \tau}$ is not considered under the assumption that traders are risk-neutral).

\section{Simulations (backtesting)}

Monte Carlo (MC) simulations that consisting of independent trials of random stock selection where each stock has an equal chance of being selected) have been conducted to compare the performance between OPS without intraday trading and OPS with the proposed method. To be specific, the number of MC trials is 100, and the number of selected stocks is $30(d=30)$. One hundred is a relatively small number for $\mathrm{MC}$ simulations, but the heavy computational burden in solving the optimisation problem in Equation (29) restricts the number of simulations. In fact, the number of $\mathrm{MC}$ simulations influences the accuracy of the numerical results, as the greater the number of $\mathrm{MC}$ simulations, the more accurate are the results. The MATLAB codes of the following experiments have been uploaded on http://www.mathworks.com/matlabcentral/fileexchange/62503 to avoid any potential ambiguity of the MC simulations.

\subsection{Assumptions for simplicity}

The following assumptions were made for simplicity:

- Assets are arbitrarily divisible (i.e. $q_{n, t}^{(j)} \in \mathbb{R}$ instead of $q_{n, t}^{(j)} \in \mathbb{Z}$ ) to avoid mixed-integer nonlinear 


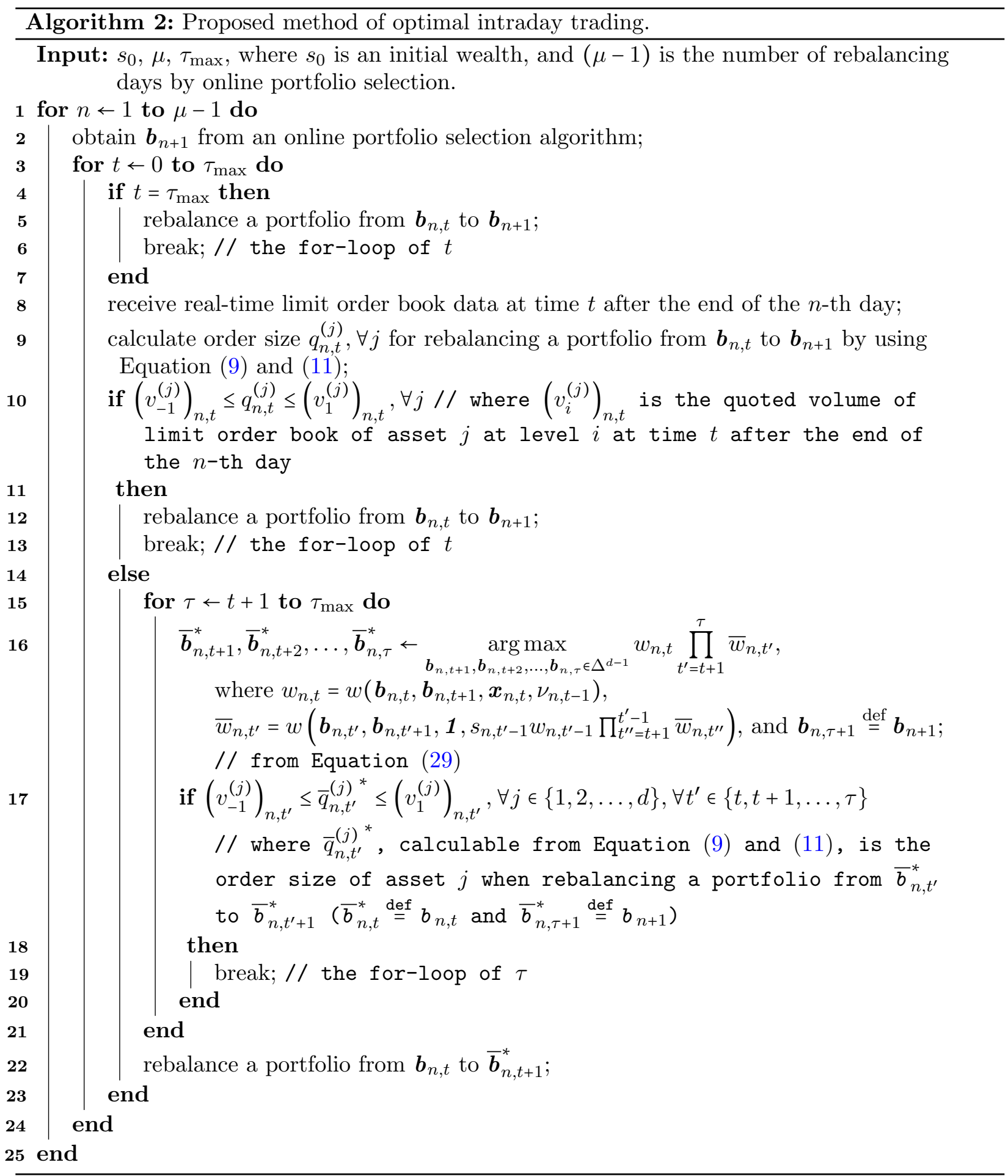

programming. ${ }^{4}$

- Hidden limit orders (HLOs), invisible in limit order books, are never submitted. ${ }^{5}$

\footnotetext{
4 Softwares of mixed-integer nonlinear programming are listed in (Bussieck and Vigerske 2011).

5 The historical quantity of HLOs can be measured by hidden volume rate, defined as the total volume of trades against hidden orders divided by the total volume of all trades. The mean of these values between 2 Jan 2014 and 30 Sep 2016 is $\{13.1 \%, 13.5 \%\}$ in the case of stocks traded on $\{$ NYSE, NASDAQ\}, respectively. The daily data of hidden volume rate is downloadable at the homepage of U.S. Securities and Exchange Commission (URL: http://www.sec.gov/opa/data/ market-structure/marketstructuredata-by-exchange.html).
} 


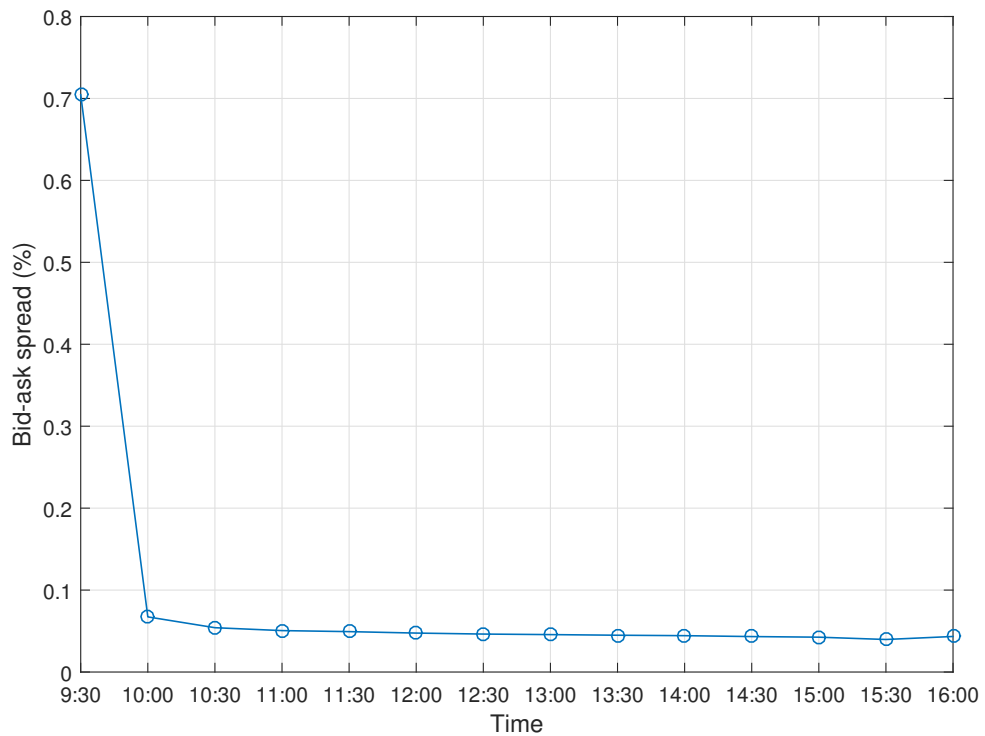

Figure 6. The median values of intraday bid-ask spreads of NASDAQ 100 Index Components (between 1 Jan 2008 and 31 Mar 2016).

- The execution of market orders by OPS at the current time $t$ does not affect the LOBs at the next time $t+1$ (this contradicts the real world but makes the backtesting feasible).

- The computation time to calculate $\boldsymbol{b}_{n, t+1}$ is zero (neither the running time between the 9th line and the 11th line in Algorithm 2 nor that between the 15th line and the 21st line in Algorithm 2 is considered).

Trading at the market opening (9:30 a.m.) was not conducted in this experiment because bid-ask spreads are much higher at the opening than mid-day or closing, as shown in Figure $6{ }^{6}$. As an bigger bid-ask spreads inevitably leads to an higher MICs : $\sum_{j=1}^{d}\left(\bar{p}\left(q_{n, t}^{(j)}\right)-m_{n, t}^{(j)}\right) q_{n, t}^{(j)}$ in Equation (8), as well as an greater proportional TCs: $c_{p} \sum_{j=1}^{d}\left(\bar{p}\left(q_{n, t}^{(j)}\right) q_{n, t}^{(j)}\right)^{+}+c_{s} \sum_{j=1}^{d}\left(-\bar{p}\left(q_{n, t}^{(j)}\right) q_{n, t}^{(j)}\right)^{+}$in Equation (8), which in turn reduces the performance of the OPS method adopted. As a result, trading starts at 10:00 a.m. in this experiment, which corresponds to time $0(t=0$; see Figure 2).

The trading interval was fixed at 30 minutes under an assumption that LOBs reverts to its normal shape within 30 minutes after the execution of market orders by OPS. This is because Xu et al. (2017) empirically showed that the intensity (or rate) of limit order submissions gradually decreases to its normal level within 30 minutes after the execution of market orders in the case of the Shanghai Stock Exchange. Consequently, $\tau_{\max }$ is set at 12 because trading starts at 10:00 a.m. instead of 9:30 a.m. and because NASDAQ regular market hours end at 4:00 p.m. ${ }^{7}$ The maximum number of intraday trades is 12 , not 13 , since the portfolio rebalancing from $\boldsymbol{b}_{n, \tau}$ to $\boldsymbol{b}_{n+1}$ is not counted as an intraday trading.

\section{2. $\quad$ The source of backtesting data}

10-level (10 levels of the ask side and 10 levels of the bid side, respectively) historical LOB data of NASDAQ 100 Index Components ${ }^{8}$ (30 components are randomly selected among the 100

6 Figure 6 corresponds to the empirical analysis by Kissell (2013, pp. 67-69) that bid-ask spreads decrease and level out after about the first 15-30 minutes for large cap stocks and after about 30-60 minutes for small cap stocks

$7 \tau_{\max }$ is 6 instead of 12 for the following NASDAQ early closing dates (NASDAQ closes at 1:00 p.m.): 3 Jul 2008, 28 Nov 2008 , 24 Dec 2008, 27 Nov 2009, 24 Dec 2009, 26 Nov 2010, 25 Nov 2011, 3 Jul 2012, 23 Nov 2012, 24 Dec 2012, 3 Jul 2013 , 29 Nov 2013, 24 Dec 2013, 3 Jul 2014, 28 Nov 2014, 24 Dec 2014, 27 Nov 2015, and 24 Dec 2015.

8 Historical NASDAQ 100 Index Components on 1 Jan 2008 was downloaded from http://siblisresearch.com/data/ historical-components-nasdaq/. 
components at each MC trial) from between 1 Jan 2008 and 31 Mar 2016 (total 2076 trading days) was downloaded from the Limit Order Book System: The Efficient Reconstructor (LOBSTER). ${ }^{9}$ The LOB data was sampled in periods of 30 minutes during NASDAQ regular market hours. The number of stock candidates used in this experiment is 83 because 17 companies were delisted before 31 Mar 2016. ${ }^{10}$ Therefore, the number of possible portfolio combinations is $\left(\begin{array}{l}83 \\ 30\end{array}\right)=3.5 \times 10^{22}$, and the portion of $\frac{100}{3.5 \times 10^{22}}=2.9 \times 10^{-21}$ is covered by the MC simulations. In addition, if accessing LOB data at greater than level 10 is required, ask price and volume at level $i \in\{11,12, \ldots\}$ are estimated as $p_{i}=p_{10}+\frac{p_{10}-p_{-1}}{10}(i-10)$ and $v_{i}=\frac{\sum_{k=1}^{10} v_{k}}{10}$, respectively. Similarly, if accessing LOB data at less than level -10 is required, bid price and volume at level $i \in\{\ldots,-12,-11\}$ are estimated as $p_{i}=p_{-10}+\frac{p_{-10}-p_{1}}{10}(-i-10)$ and $v_{i}=\frac{\sum_{k=1}^{10} v_{-k}}{10}$, respectively.

When calculating the relative price of asset $j$ between time $\tau$ after the end of the $(n-1)$-th day and the end of the $n$-th day, cash dividends, stock dividends, and stock splits were considered as

$$
x_{n, 0}^{(j)}=\frac{m_{n, 0}^{(j)} \frac{a_{n, \tau_{\max }}^{(j)}}{g_{n, \tau_{\max }}^{(j)}}}{m_{n-1, \tau}^{(j)} \frac{a_{n-1, \tau_{\max }}^{(j)}}{g_{n-1, \tau_{\max }}^{(j)}}},
$$

where $m_{n, t}^{(j)}$ is the mid-price of asset $j$ at time $t$ after the end of the $n$-th day from LOBSTER, and $\left\{g_{n, \tau_{\max }}^{(j)}, a_{n, \tau_{\max }}^{(j)}\right\}$ (the subscript $n, \tau_{\max }$ indicates time $\tau_{\max }$ after the end of the $n$-th day) is the \{closing, adjusted closing $\}$ price of asset $j$ of the $(n+1)$-th day, not the $n$-th day (a day ends after or at the market opening in this paper; see Figure 2), from Yahoo Finance.

\subsection{Performance comparison among online portfolio selection methods without transaction costs}

The performance (annualised return) of the OPS methods in Table 2 without TCs $\left(c_{p}=0, c_{s}=0\right.$, and zero MICs) is compared in Table 3 and Figure $7^{11}$, where all the OPS methods rebalanced a portfolio at 10:00 a.m. on every U.S. trading day. In particular, the unpaired two-sample $t$-tests with unequal variances (hereafter we simply referred to as $t$-tests), whose null hypothesis is that the data in two groups comes from independent random samples from normal distributions with equal means but different variances, were performed to compare the performance between i) a buy-and-hold $(\mathrm{B} \& \mathrm{H})$ strategy with the initial portfolio $\boldsymbol{b}_{1}=[1 / d 1 / d \ldots 1 / d]^{\mathrm{T}}$ and ii) the OPS methods listed in Table 2. Also, the normality assumption of the $t$-test was confirmed by the Jarque-Bera (JB) test with the significance level of 0.05 as shown in Table 3 except OLMAR1, OLMAR2, and BK. The standard deviation in Table 3 can be interpreted as the sensitivity of the annualised return to the random stock selection. USCRP and OLMAR1 are the least and the most sensitive, respectively.

The $t$-tests provide the answer as to whether the performance difference of the two methods is significant or whether it is due to random fluctuations (Simon 2013, p. 631). To be specific, the $p$-value of the $t$-test is interpreted as the probability that a difference in the mean values would be obtained, given that the population means of the two methods are equivalent (the $p$-value is not equal to the probability that the population means are equivalent) (Simon 2013, p. 635). Hence, if the $p$-value of the $t$-test is less than a significance level, the performance difference is significant. All

9 LOBSTER (https://lobsterdata.com/) has LOB data from 27 Jun 2007 to the present, and the LOB data of LOBSTER does not include hidden LOBs (Huang and Polak 2011, Table 1).

10 ALTR, AMLN, BEAS, BRCM, CEPH, DELL, FMCN, GENZ, JAVA, LEAP, NIHD, PETM, SIAL, TLAB, and VMED were acquired by other companies, and FWLT and MIICF were voluntarily delisted from NASDAQ.

${ }^{11}$ MATLAB programs of OPS by Li and Hoi (2015, Appendix A) were used to obtain Table 3 and Figure 7 
Table 2. A list of online portfolio selection (OPS) strategies.

\begin{tabular}{|c|c|c|c|}
\hline Category & Author(s) & OPS strategy & Abbreviation \\
\hline \multirow{5}{*}{ Follow the winner } & Cover $(1991)$ & Universal portfolio & UP \\
\hline & Helmbold et al. (1998) & Exponential gradient & EG \\
\hline & Borodin et al. (2000) & Markov of order zero & M0, T0 ${ }^{\mathrm{a}}$ \\
\hline & Agarwal et al. (2006) & Online Newton step & ONS \\
\hline & Kozat and Singer (2011) & $\begin{array}{l}\text { Universal semi-constant rebal- } \\
\text { anced portfolio }\end{array}$ & USCRP \\
\hline \multirow{4}{*}{ Follow the loser } & Borodin et al. (2004) & Anti-correlation & ANTICOR, ANTICOR_ANTICOR ${ }^{b}$ \\
\hline & Li and Hoi (2012) & Online moving average reversion & OLMAR1, OLMAR2 ${ }^{\mathrm{C}}$ \\
\hline & Li et al. (2012) & Passive aggressive mean reversion & PAMR, PAMR1, PAMR2 $2^{\mathrm{d}}$ \\
\hline & Li et al. (2013) & $\begin{array}{l}\text { Confidence weighted mean rever- } \\
\text { sion }\end{array}$ & CWMR_VAR, CWMR_STDEV ${ }^{\mathrm{e}}$ \\
\hline \multirow[t]{3}{*}{ Pattern matching } & Györfi et al. (2006) & $\begin{array}{l}\text { Nonparametric kernel-based log- } \\
\text { optimal }\end{array}$ & BK \\
\hline & Györfi et al. (2008) & $\begin{array}{l}\text { Nonparametric nearest neighbour } \\
\text { log-optimal }\end{array}$ & $\mathrm{BNN}$ \\
\hline & Li et al. (2011) & $\begin{array}{l}\text { Correlation-driven nonparametric } \\
\text { learning }\end{array}$ & CORN, CORNU, CORNK ${ }^{\mathrm{f}}$ \\
\hline
\end{tabular}

a T0 considers historical market vectors $\boldsymbol{x}_{1}, \boldsymbol{x}_{2}, \ldots, \boldsymbol{x}_{n}$ fully, while M0 does not.

${ }^{\mathrm{b}}$ ANTICOR_ANTICOR is the twice compounded algorithm of ANTICOR.

${ }^{\mathrm{c}}$ OLMAR1 uses a simple (equally weighted) moving average, while OLMAR2 uses an exponential (exponentially weighted) moving average.

dPAMR1 added a slack variable $\xi$ to the objective function of PAMR, and PAMR2 added $\xi^{2}$.

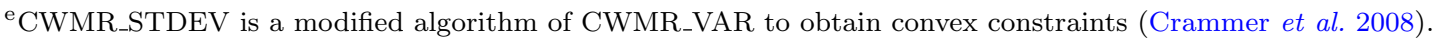

${ }^{\mathrm{f}} \mathrm{CORN}$ : each expert has a weight proportional to its historical performance; CORNU: all experts have the same weight; CORNK: only the K-best experts have weights. 
Table 3. Statistics of annualised returns of different online portfolio selection methods without transaction costs $\left(c_{p}=0, c_{s}=0\right.$, and zero market impact costs).

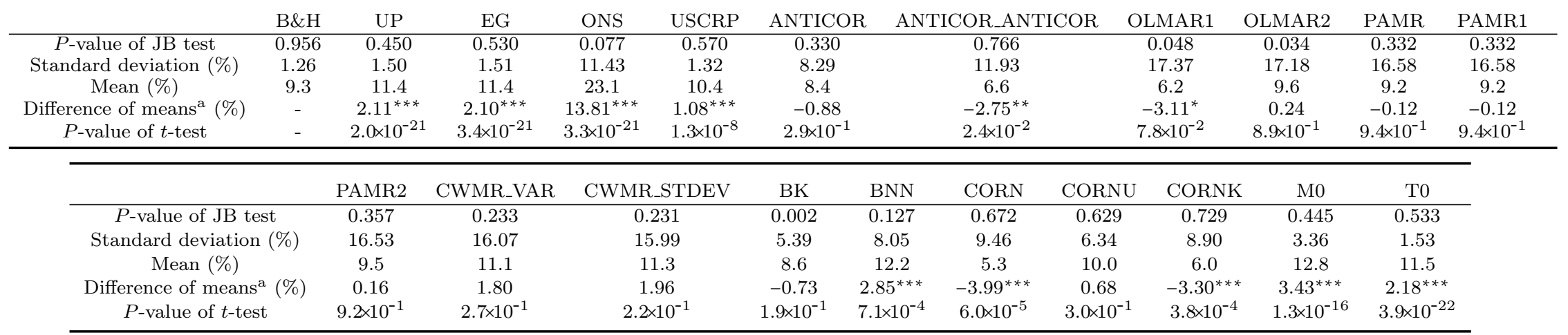

${ }^{\text {a }}$ Difference equals average annualised return of the corresponding OPS method minus that of buy-and-hold $(\mathrm{B} \& \mathrm{H}) .{ }^{*} p<0.1 ;{ }^{* *} p<0.05 ;{ }^{* * *} p<0.01$.

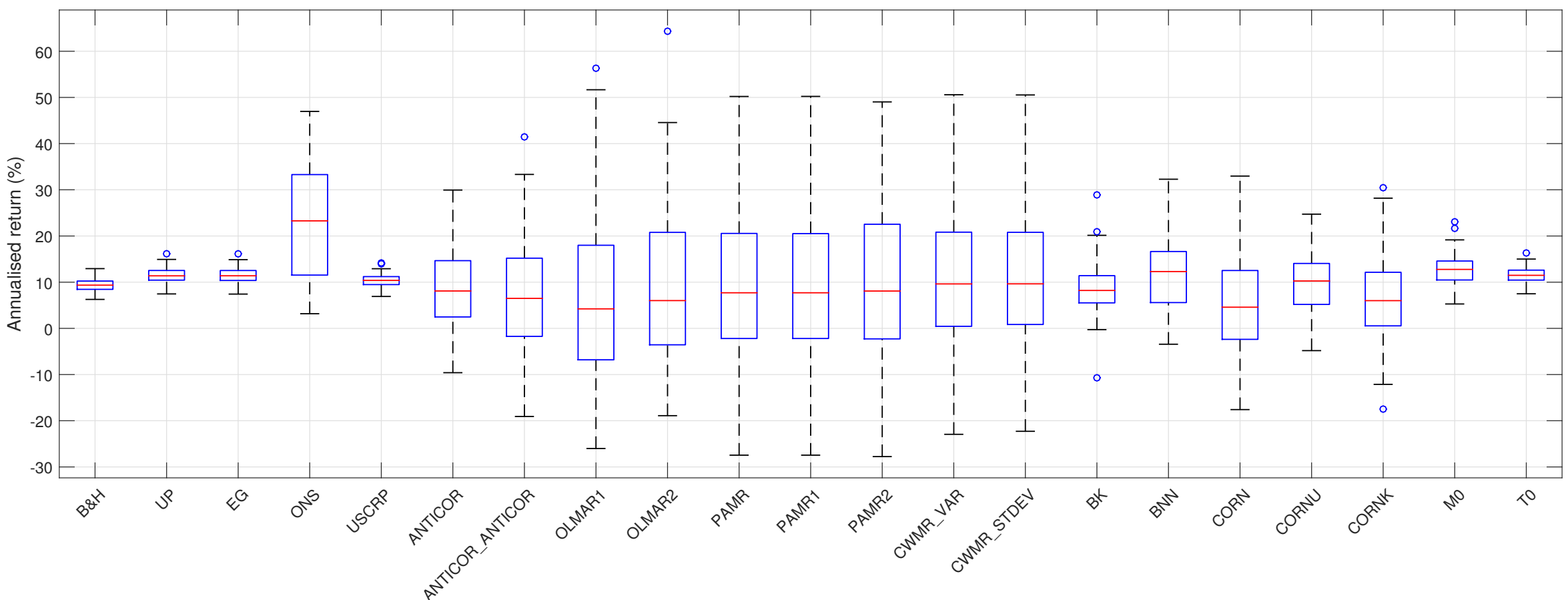

Figure 7. Box plots of annualised returns of different online portfolio selection methods without transaction costs $\left(c_{p}=0, c_{s}=0\right.$, and zero market impact costs). 
the OPS methods of follow the winner are highly superior to B\&H as shown in Table 3. In contrast, all the OPS methods of follow the loser and pattern matching except BNN are not superior to B\&H, and some of them are inferior to $\mathrm{B} \& \mathrm{H}$ (i.e. the differences of means are negative with low $p$-values in Table 3). However, this should not be interpreted that the OPS methods of follow the loser and pattern matching are always inferior to those of follow the winner, as the NASDAQ bull market between 2009 and 2016 (see Figure 8) was unfavourable to the OPS methods of follow the loser.

The performance of each OPS method without TCs and intraday trading in Table 3 and Figure 7 can be considered as the upper limit of the performance with TCs and intraday trading. This is because OPS carries out a long-term investment for every period at time $t=0$, while the proposed method of intraday trading absorbs the shock to the market whenever OPS rebalances a portfolio. In other words, the proposed method minimises the performance gap between OPS without TCs and OPS with TCs, but no additional profits made.

\subsection{Performance comparison between online portfolio selection methods with and without the proposed method}

Based on the $p$-values of the $t$-tests in Table 3, T0, UP, and ONS have been selected as the best three OPS methods for the comparison. We compare the performance of each OPS method without and with the proposed method (PM henceforth) using Algorithm 2. All of these three methods increase the relative weights of more successful assets in the past periods (i.e. following the winner), as the portfolio vector of each algorithm in the next period $\boldsymbol{b}_{n+1}\left(\right.$ or $\left.b_{n+1}^{(j)}\right)$ is

- T0: $b_{n+1}^{(j)}=\frac{c_{n}^{(j)}+\beta}{d \beta+\sum_{j^{\prime}=1}^{d} c_{n}^{\left(j^{\prime}\right)}}$, where $c_{n}^{(j)}=c_{n-1}^{(j)}+\log _{2}\left(1+x_{n}^{(j)}\right)$, and $\beta \in[0, \infty)$ is a parameter;

- UP: $\boldsymbol{b}_{n+1}=\frac{\int_{\Delta^{d-1}} \boldsymbol{b} s_{n}\left(\boldsymbol{b}, \boldsymbol{x}_{1: n}\right) d \boldsymbol{b}}{\int_{\Delta^{d-1}} s_{n}\left(\boldsymbol{b}, \boldsymbol{x}_{1: n}\right) d \boldsymbol{b}}$, where, $s_{n}\left(\boldsymbol{b}, \boldsymbol{x}_{1: n}\right)=s_{0} \prod_{i=1}^{n}\left\langle\boldsymbol{b}, \boldsymbol{x}_{i}\right\rangle$ is wealth at the end of the $n$-th period with an initial wealth $s_{0}$ (the integral can be calculated numerically by using MC methods (Ishijima 2001));

- ONS: $\boldsymbol{b}_{n+1}=\underset{\boldsymbol{b} \in \Delta^{d-1}}{\arg \max }\left(\sum_{i=1}^{n} \ln \left\langle\boldsymbol{b}, \boldsymbol{x}_{i}\right\rangle-\frac{\beta}{2}\|\boldsymbol{b}\|^{2}\right)$, where $\beta \in[0, \infty)$ is a trade-off parameter between the follow the winner term $\sum_{i=1}^{n} \ln \left\langle\boldsymbol{b}, \boldsymbol{x}_{i}\right\rangle$ and the regularisation term $\|\boldsymbol{b}\|^{2}$.

Transaction costs (TCs), consisting of proportional TCs and MICs, has been calculated whenever a portfolio was rebalanced for each case. More specifically, for the case of OPS without using the PM TCs were calculated at 10:00 a.m. on every trading day, whereas they were calculated not only at 10:00 a.m. but also between 10:30 a.m. and 4:00 p.m. in the period of 30 minutes on every trading day for the case of OPS using the PM.

The range of the proportional TC rate was set as $c_{p}=0$ and $0.00218 \% \leq c_{s} \leq 0.5 \%$ as securities transaction tax rates in most of the G20 countries varied between $0.1 \%$ and $0.5 \%$ (Matheson 2011) - in the United States in 2016, they were $c_{p}=0$ and $c_{s}=0.218$ basis points. ${ }^{12}$

If initial wealth $s_{0}$ is as small as USD 100,000, the performance difference between OPS without and with the PM is not statistically significant, as shown in Table 4 and Figure 11. The statistical significance can be determined by both the sign and $p$-value of the difference of means of annualised return between without and with the PM. A positive difference with low $p$-value indicates the PM is useful. However, the lowest $p$-value (when using ONS at $c_{s}=0.00218 \%$ ) is as high as 0.641 as shown in Table 4, which means the PM is not useful for small-sized funds.

However, the PM is useful when initial wealth $s_{0}$ is as large as USD 1,000,000 as shown in Table 5 and Figure 12. In particular, the performance difference between ONS without and with the PM is

\footnotetext{
12 Order making fiscal year 2016 annual adjustments to transaction fee rates, U.S. Securities and Exchange Commission [Release No. 34-76848/7 Jan 2016].
} 
statistically significant. In addition, the performance difference between without and with the PM varies by OPS method. ONS makes a greater performance gap than T0 and UP. This is because ONS is a more dynamic investment strategy than T0 and UP as shown in Figure 9; i.e. the PM has more opportunities to reduce TCs when OPS tries to cause the higher TCs.

The performance difference between without and with the PM (the difference of means in Table 4 and Table 5) is less significant when $c_{s}$ is higher. This is because proportional TCs, $c_{p} \sum_{j=1}^{d}\left(\bar{p}\left(q_{n, t}^{(j)}\right) q_{n, t}^{(j)}\right)^{+}+c_{s} \sum_{j=1}^{d}\left(-\bar{p}\left(q_{n, t}^{(j)}\right) q_{n, t}^{(j)}\right)^{+}$in Equation (8), are more dominant compared to MICs, $\sum_{j=1}^{d}\left(\bar{p}\left(q_{n, t}^{(j)}\right)-m_{n, t}^{(j)}\right) q_{n, t}^{(j)}$ in Equation (8), when $c_{p}$ or $c_{s}$ is the greater. Even if a large market order is divided into consecutive intraday market orders by the PM, proportional TCs with the $\mathrm{PM}$ are as large as those without the PM. Consequently, the PM is more useful with the lower $c_{p}$ and $c_{s}$.

\subsection{Graphical comparisons}

Figure 8 shows gross wealth $s_{n}$ of a portfolio consisting of six stocks with an initial wealth of a million USD. The PM of intraday trading has little value in the case of T0 and UP as shown in Figure 8(a) and Figure 8(b). However, the PM works well when ONS is used as shown in Figure 8(c). These correspond to the performance difference without and with the PM in Table 5 and Figure 12.

Figure 9 shows the proportion of the portfolio (the portfolio vector $\boldsymbol{b}_{n}$ ) that made the gross wealth plots in Figure 8, in the form of area plots $\left(\boldsymbol{b}_{n}\right.$ is independent of the usage of an intraday trading algorithm). The portfolio vector of $\mathrm{B} \& \mathrm{H}$ changes over time as the prices of assets also change over time as shown in Figure 9(a). T0 generates almost constant portfolio $\boldsymbol{b}_{n}=\boldsymbol{b}_{1}$ as shown in Figure 9(b), and UP generates rougher portfolio weights over time than those of T0 but smoother changes than those of B\&H as shown in Figure 9(c). ONS makes the most abrupt changes of portfolio weights over time as shown in Figure 9(d).

Figure 10 shows how much the PM can decrease TCs $c_{n}$, consisting of both proportional TCs and MICs, when following the gross wealth in Figure 8. TC reduction by the PM is not significant in the case of T0 and UP as shown in Figure 10(a) and Figure 10(b) since both T0 and UP do not trade stocks too much. On the contrary, TCs are reduced greatly in the case of a high-volume trading algorithm like ONS as shown in Figure 10(c). 


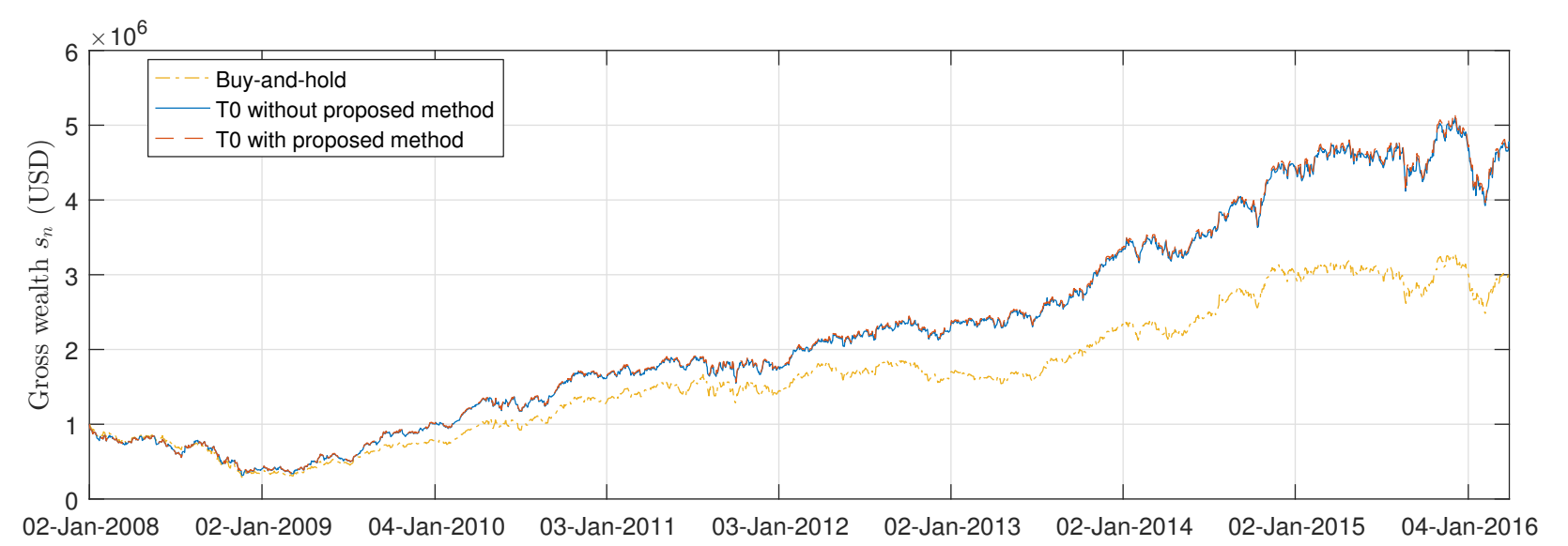

(a) T0.

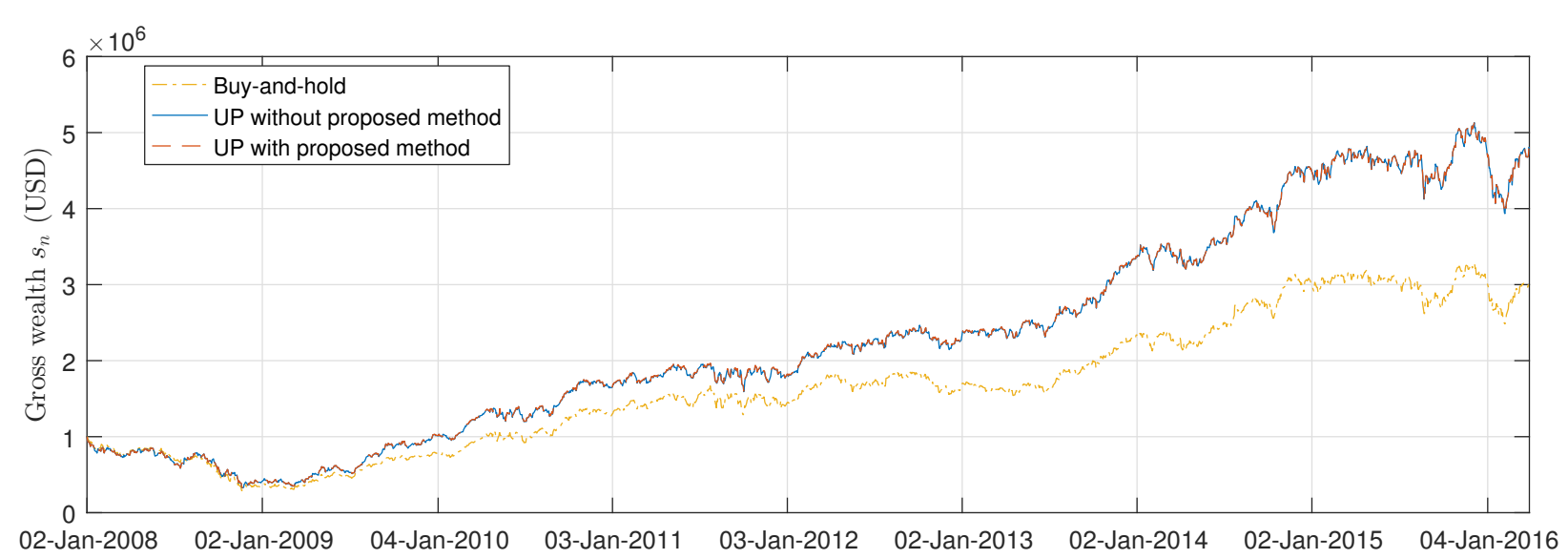

(b) UP.

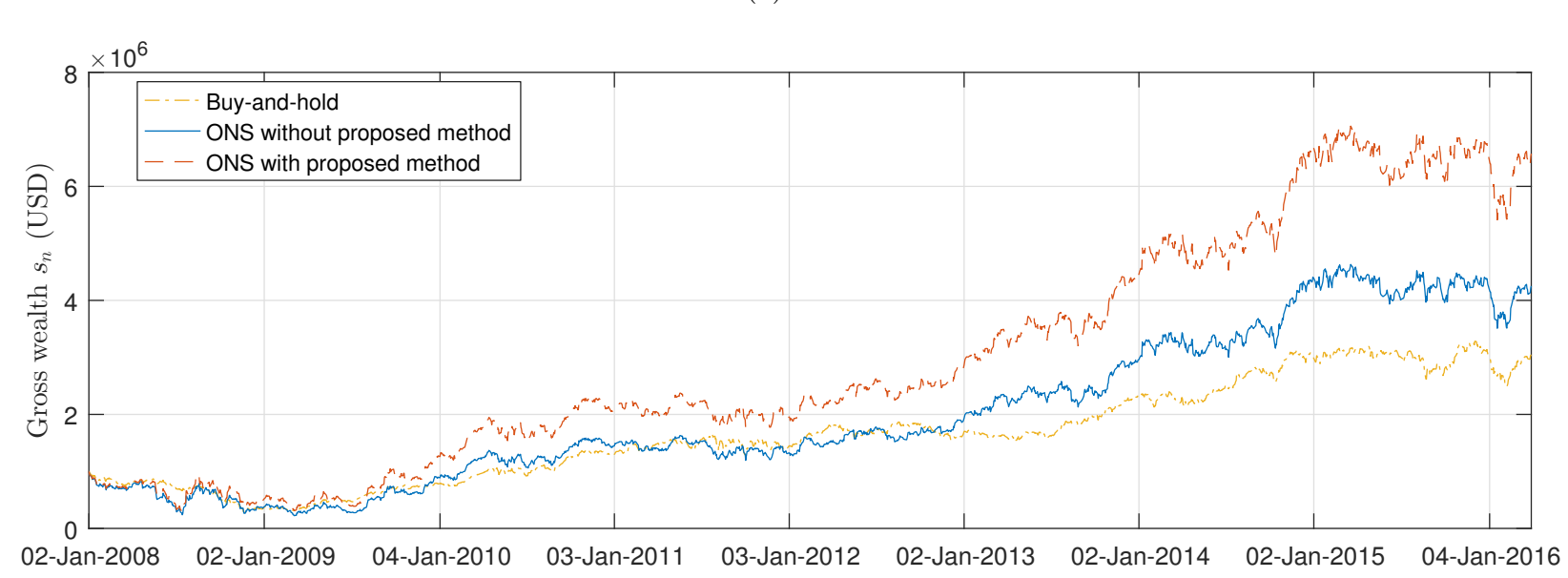

(c) ONS.

Figure 8. Gross wealth over time when the portfolio consists of AAPL, BIDU, EXPE, QVCA, UAL, and VRSN

$\left(s_{0}=10^{6} \mathrm{USD}, c_{p}=0, c_{s}=0.00218 \%\right)$. 


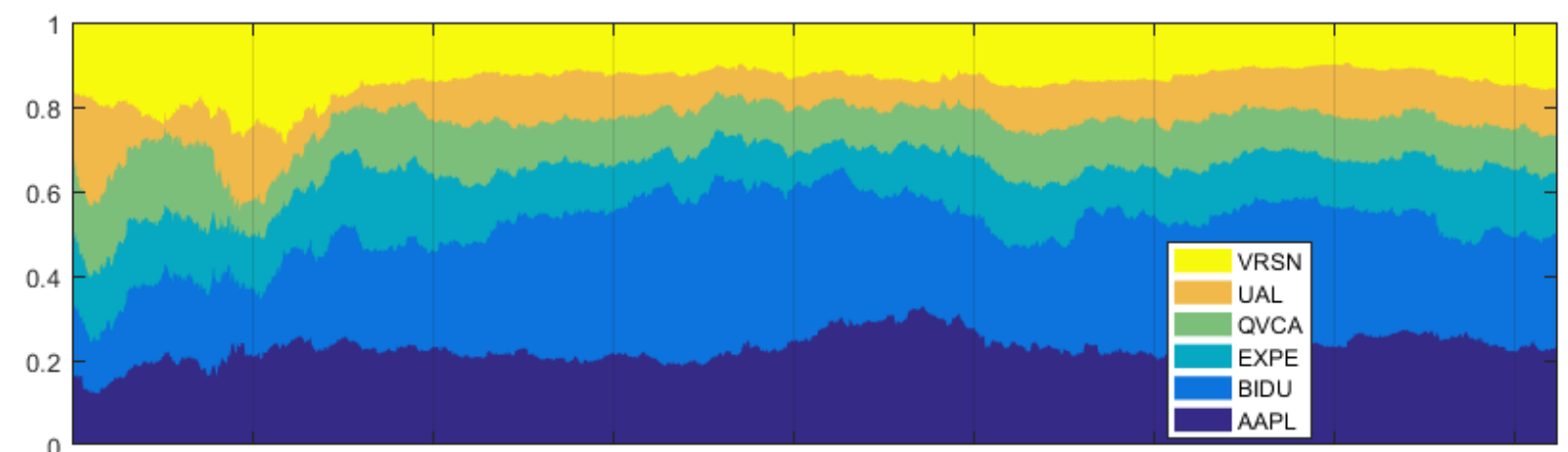

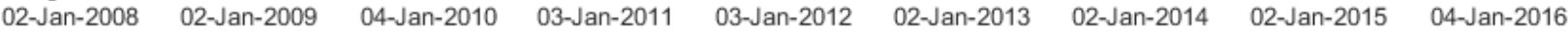
(a) Buy-and-hold $(\mathrm{B} \& \mathrm{H})$.

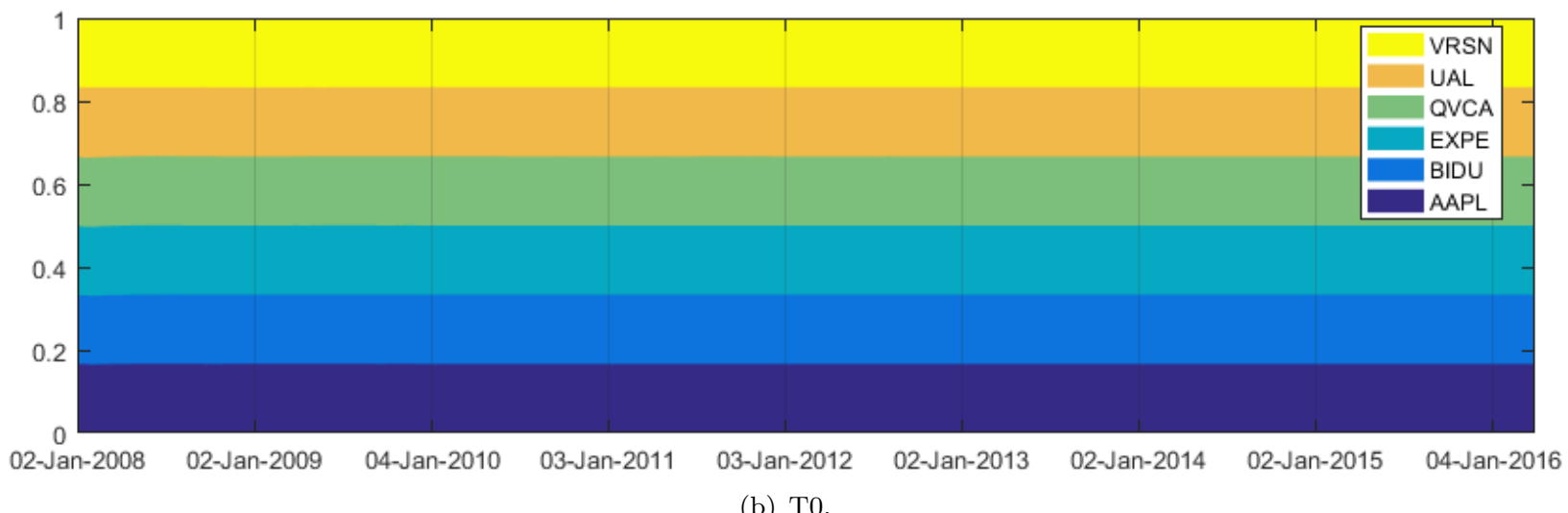

(b) T0.

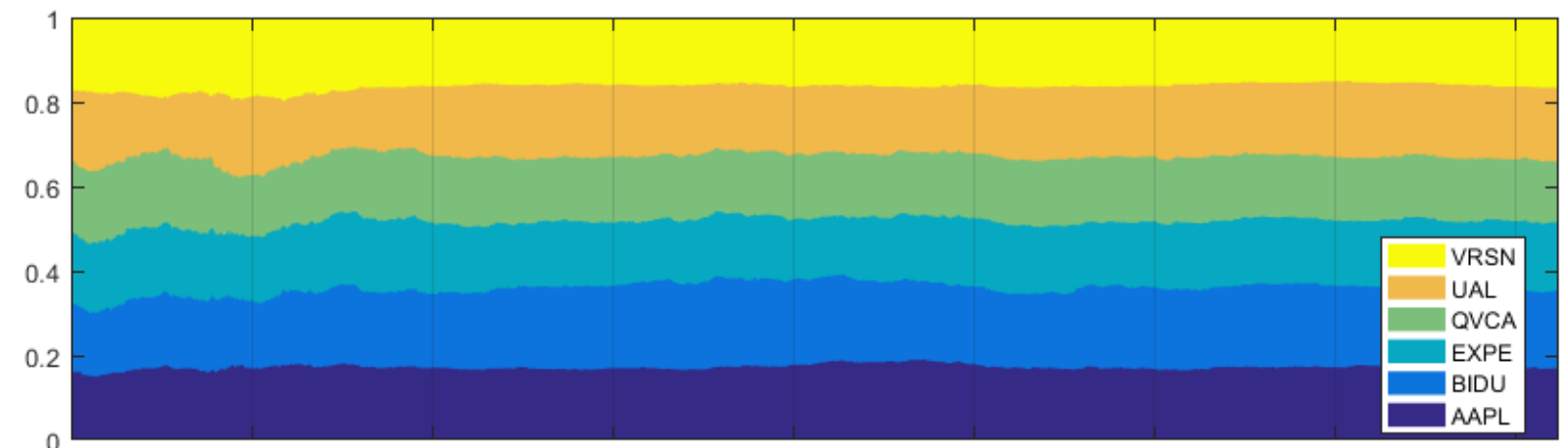

02-Jan-2008 $\quad$ 02-Jan-2009 $\quad$ 04-Jan-2010 $\quad$ 03-Jan-2011 $\quad$ 03-Jan-2012 $\quad$ 02-Jan-2013 $\quad$ 02-Jan-2014 $\quad$ 02-Jan-2015 $04-J a n-2016$ (c) UP.

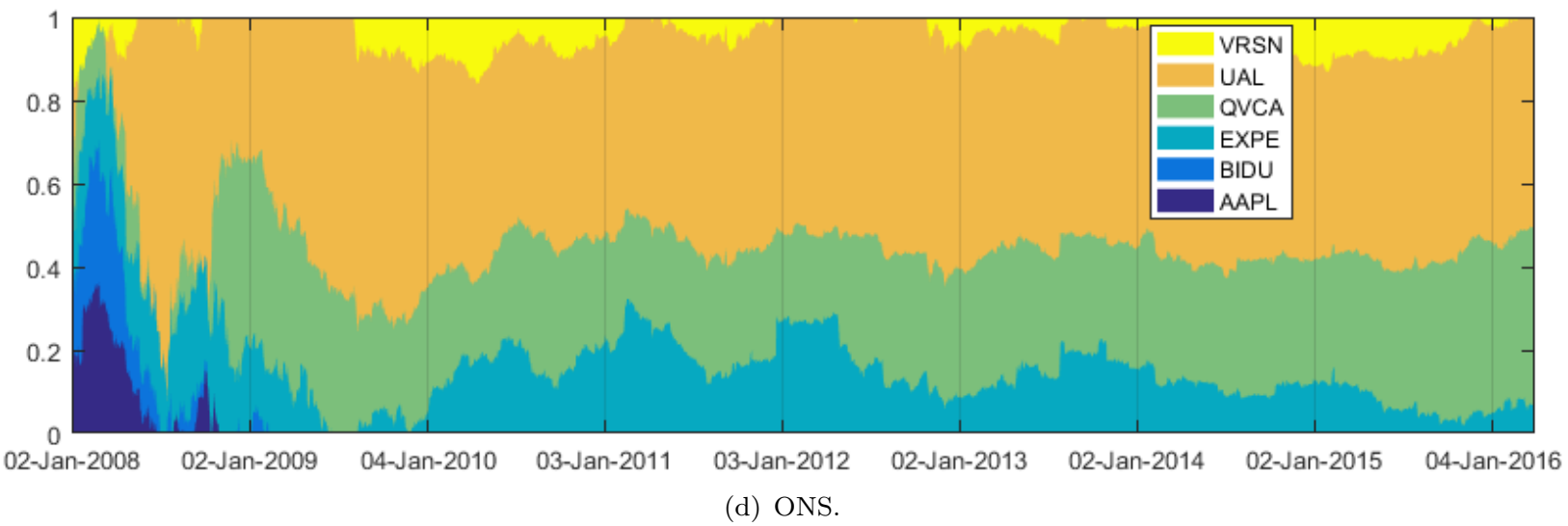

Figure 9. The proportion of portfolio over time. 
Table 4. Statistics of annualised returns of different online portfolio selection methods without and with proposed method $(\mathrm{PM})\left(c_{p}=0, s_{0}=10^{5} \mathrm{USD}\right)$

\begin{tabular}{|c|c|c|c|c|c|c|c|c|c|c|c|c|c|}
\hline$c_{s}(\%)$ & $\mathrm{B} \& \mathrm{H}$ & $\begin{array}{c}0.00218 \\
\text { T0 } \\
\text { w/o PM }\end{array}$ & $\begin{array}{c}0.00218 \\
\text { T0 } \\
\mathrm{w} / \mathrm{PM}\end{array}$ & $\begin{array}{c}0.00218 \\
\text { UP } \\
\text { w/o PM }\end{array}$ & $\begin{array}{c}0.00218 \\
\text { UP } \\
\text { w/ PM }\end{array}$ & $\begin{array}{c}0.00218 \\
\text { ONS } \\
\text { w/o PM }\end{array}$ & $\begin{array}{c}0.00218 \\
\text { ONS } \\
\mathrm{w} / \mathrm{PM}\end{array}$ & $\begin{array}{c}0.16667 \\
\text { T0 } \\
\text { w/o PM }\end{array}$ & $\begin{array}{c}0.16667 \\
\text { T0 } \\
\text { w/ PM }\end{array}$ & $\begin{array}{c}0.16667 \\
\text { UP } \\
\text { w/o PM }\end{array}$ & $\begin{array}{c}0.16667 \\
\text { UP } \\
\text { w/ PM }\end{array}$ & $\begin{array}{c}0.16667 \\
\text { ONS } \\
\text { w/o PM }\end{array}$ & $\begin{array}{c}0.16667 \\
\text { ONS } \\
\text { w/ PM }\end{array}$ \\
\hline$P$-value of JB test & 0.956 & 0.594 & 0.617 & 0.509 & 0.503 & 0.076 & 0.082 & 0.603 & 0.585 & 0.519 & 0.514 & 0.073 & 0.079 \\
\hline Standard deviation (\%) & 1.26 & 1.53 & 1.53 & 1.50 & 1.50 & 10.93 & 11.22 & 1.53 & 1.53 & 1.50 & 1.50 & 10.88 & 11.12 \\
\hline Mean $(\%)$ & 9.3 & 11.3 & 11.3 & 11.2 & 11.2 & 21.3 & 22.1 & 11.0 & 11.0 & 11.0 & 11.0 & 20.1 & 20.7 \\
\hline Difference of means ${ }^{\mathrm{a}}(\%)$ & - & $1.97^{* * *}$ & $1.97^{* * *}$ & $1.92^{* * *}$ & $1.92^{* * *}$ & $11.99^{* * *}$ & $12.73^{* * *}$ & $1.69^{* * *}$ & $1.69^{* * *}$ & $1.65^{* *}$ & $1.65^{* * *}$ & $10.72^{* * *}$ & $11.36^{* * *}$ \\
\hline$P$-value of $t$-test & - & $4.6 \times 10^{-19}$ & $4.9 \times 10^{-19}$ & $1.3 \times 10^{-18}$ & $1.3 \times 10^{-18}$ & $8.9 \times 10^{-19}$ & $1.3 \times 10^{-19}$ & $4.9 \times 10^{-15}$ & $4.0 \times 10^{-15}$ & $8.2 \times 10^{-15}$ & $8.2 \times 10^{-15}$ & $2.4 \times 10^{-16}$ & $4.1 \times 10^{-17}$ \\
\hline Difference of means ${ }^{\mathrm{b}}(\%)$ & - & \multirow{2}{*}{\multicolumn{2}{|c|}{$\begin{array}{l}-0.00 \\
0.990\end{array}$}} & \multirow{2}{*}{\multicolumn{2}{|c|}{$\begin{array}{c}0.00 \\
0.999\end{array}$}} & \multirow{2}{*}{\multicolumn{2}{|c|}{$\begin{array}{r}0.73 \\
0.641\end{array}$}} & \multirow{2}{*}{\multicolumn{2}{|c|}{$\begin{array}{c}0.01 \\
0.976\end{array}$}} & \multirow{2}{*}{\multicolumn{2}{|c|}{$\begin{array}{r}0.00 \\
0.099\end{array}$}} & \multicolumn{2}{|c|}{0.63} \\
\hline$P$-value of $t$-test & - & & & & & & & & & & & & \\
\hline
\end{tabular}

\begin{tabular}{|c|c|c|c|c|c|c|c|c|c|c|c|c|}
\hline$c_{s}(\%)$ & $\begin{array}{c}0.33333 \\
\text { T0 } \\
\text { w/o PM }\end{array}$ & $\begin{array}{c}0.33333 \\
\text { T0 } \\
\mathrm{w} / \mathrm{PM}\end{array}$ & $\begin{array}{c}0.33333 \\
\text { UP } \\
\text { w/o PM }\end{array}$ & $\begin{array}{c}0.33333 \\
\text { UP } \\
\text { w/ PM }\end{array}$ & $\begin{array}{c}0.33333 \\
\text { ONS } \\
\text { w/o PM }\end{array}$ & $\begin{array}{c}0.33333 \\
\text { ONS } \\
\mathrm{w} / \mathrm{PM}\end{array}$ & $\begin{array}{c}0.5 \\
\text { T0 } \\
\text { w/o PM }\end{array}$ & $\begin{array}{c}0.5 \\
\text { T0 } \\
\mathrm{w} / \mathrm{PM}\end{array}$ & $\begin{array}{c}0.5 \\
\text { UP } \\
\text { w/o PM }\end{array}$ & $\begin{array}{c}0.5 \\
\text { UP } \\
\mathrm{w} / \mathrm{PM}\end{array}$ & $\begin{array}{c}0.5 \\
\text { ONS } \\
\text { w/o PM }\end{array}$ & $\begin{array}{c}0.5 \\
\text { ONS } \\
\mathrm{w} / \mathrm{PM}\end{array}$ \\
\hline$P$-value of JB test & 0.612 & 0.595 & 0.529 & 0.524 & 0.070 & 0.075 & 0.621 & 0.606 & 0.539 & 0.534 & 0.067 & 0.073 \\
\hline Standard deviation (\%) & 1.53 & 1.53 & 1.50 & 1.50 & 10.83 & 11.08 & 1.53 & 1.53 & 1.50 & 1.50 & 10.78 & 11.02 \\
\hline Mean (\%) & 10.7 & 10.7 & 10.7 & 10.7 & 18.8 & 19.4 & 10.4 & 10.4 & 10.4 & 10.4 & 17.5 & 18.1 \\
\hline Difference of means ${ }^{\mathrm{a}}(\%)$ & $1.40^{* * *}$ & $1.40^{* * *}$ & $1.38^{* * *}$ & $1.38^{* * *}$ & $9.45^{\star * *}$ & $10.08^{* * *}$ & $1.11^{* * *}$ & $1.11^{* * *}$ & $1.11^{* * *}$ & $1.11^{* * *}$ & $8.18^{* * *}$ & $8.78^{* * *}$ \\
\hline$P$-value of $t$-test & $3.0 \times 10^{-11}$ & $2.6 \times 10^{-11}$ & $3.2 \times 10^{-11}$ & $3.2 \times 10^{-11}$ & $7.3 \times 10^{-14}$ & $1.1 \times 10^{-14}$ & $7.3 \times 10^{-8}$ & $6.3 \times 10^{-8}$ & $5.4 \times 10^{-8}$ & $5.3 \times 10^{-8}$ & $2.0 \times 10^{-11}$ & $3.2 \times 10^{-12}$ \\
\hline Difference of means ${ }^{\mathrm{b}}(\%)$ & \multicolumn{2}{|c|}{0.01} & \multicolumn{2}{|c|}{0.00} & \multicolumn{2}{|c|}{0.63} & \multicolumn{2}{|c|}{0.01} & \multicolumn{2}{|c|}{0.00} & \multicolumn{2}{|c|}{0.60} \\
\hline$P$-value of $t$-test & \multicolumn{2}{|c|}{0.977} & \multicolumn{2}{|c|}{0.999} & \multicolumn{2}{|c|}{0.686} & \multicolumn{2}{|c|}{0.977} & \multicolumn{2}{|c|}{0.999} & \multicolumn{2}{|c|}{0.699} \\
\hline
\end{tabular}

${ }^{\text {a }}$ Difference equals average annualised return of corresponding OPS method minus that of buy-and-hold $(\mathrm{B} \& \mathrm{H}) .{ }^{*} p<0.1 ;{ }^{* *} p<0.05 ;{ }^{* * *} p<0.01$.

${ }^{\mathrm{b}}$ Difference equals average annualised return of corresponding OPS method with proposed method (PM) minus that without $\mathrm{PM} .{ }^{*} p<0.1 ;{ }^{* *} p<0.05 ;{ }^{* * *} p<0.01$.

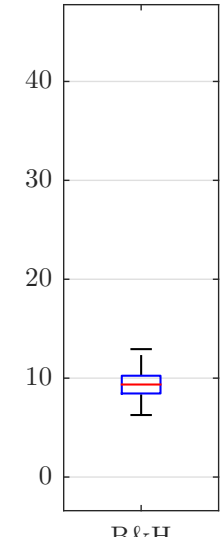

$B \& \mathrm{H}$

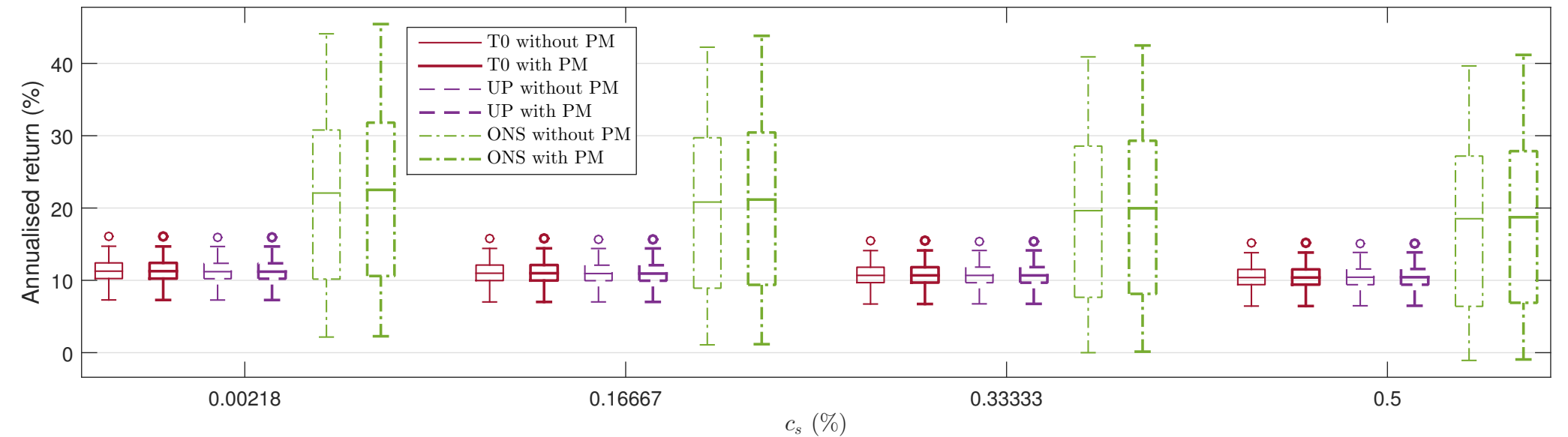

Figure 11. Box plots of annualised returns of different online portfolio selection methods without and with the proposed method $(\mathrm{PM})\left(c_{p}=0, s_{0}=10^{5} \mathrm{USD}\right)$. 
Table 5. Statistics of annualised returns of different online portfolio selection methods without and with proposed method $(\mathrm{PM})\left(c_{p}=0, s_{0}=10^{6} \mathrm{USD}\right)$

\begin{tabular}{|c|c|c|c|c|c|c|c|c|c|c|c|c|c|}
\hline$c_{s}(\%)$ & $\mathrm{BnH}$ & $\begin{array}{c}0.00218 \\
\text { T0 } \\
\text { w/o PM }\end{array}$ & $\begin{array}{c}0.00218 \\
\text { T0 } \\
\mathrm{w} / \mathrm{PM}\end{array}$ & $\begin{array}{c}0.00218 \\
\text { UP } \\
\text { w/o PM }\end{array}$ & $\begin{array}{c}0.00218 \\
\text { UP } \\
\text { w/ PM }\end{array}$ & $\begin{array}{c}0.00218 \\
\text { ONS } \\
\mathrm{w} / \mathrm{o} \mathrm{PM}\end{array}$ & $\begin{array}{c}0.00218 \\
\text { ONS } \\
\mathrm{w} / \mathrm{PM}\end{array}$ & $\begin{array}{c}0.16667 \\
\text { T0 } \\
\text { w/o PM }\end{array}$ & $\begin{array}{c}0.16667 \\
\text { T0 } \\
\text { w/ PM }\end{array}$ & $\begin{array}{c}0.16667 \\
\text { UP } \\
\text { w/o PM }\end{array}$ & $\begin{array}{c}0.16667 \\
\text { UP } \\
\text { w/ PM }\end{array}$ & $\begin{array}{c}0.16667 \\
\text { ONS } \\
\text { w/o PM }\end{array}$ & $\begin{array}{c}0.16667 \\
\text { ONS } \\
\text { w/ PM }\end{array}$ \\
\hline$P$-value of JB test & 0.956 & 0.643 & 0.552 & 0.511 & 0.487 & 0.059 & 0.090 & 0.651 & 0.564 & 0.520 & 0.503 & 0.057 & 0.086 \\
\hline Standard deviation (\%) & 1.26 & 1.53 & 1.54 & 1.50 & 1.51 & 10.12 & 11.23 & 1.53 & 1.53 & 1.50 & 1.50 & 10.10 & 11.17 \\
\hline Mean $(\%)$ & 9.3 & 11.3 & 11.3 & 11.3 & 11.3 & 18.6 & 22.1 & 11.0 & 11.0 & 11.0 & 11.0 & 17.5 & 20.8 \\
\hline Difference of means ${ }^{\mathrm{a}}(\%)$ & - & $1.95^{* * *}$ & $2.00^{* * *}$ & $1.93^{* * *}$ & $1.93^{* * *}$ & $9.24^{* * *}$ & $12.74^{* * *}$ & $1.66^{* *}$ & $1.71^{* * *}$ & $1.66^{* *}$ & $1.66^{* * *}$ & $8.12^{* * *}$ & $11.51^{* * *}$ \\
\hline$P$-value of $t$-test & - & $9.2 \times 10^{-19}$ & $2.0 \times 10^{-19}$ & $8.5 \times 10^{-19}$ & $8.8 \times 10^{-19}$ & $9.8 \times 10^{-15}$ & $1.3 \times 10^{-19}$ & $9.2 \times 10^{-15}$ & $2.4 \times 10^{-15}$ & $5.5 \times 10^{-15}$ & $5.9 \times 10^{-15}$ & $2.2 \times 10^{-12}$ & $2.5 \times 10^{-17}$ \\
\hline Difference of means ${ }^{\mathrm{b}}(\%)$ & - & \multirow{2}{*}{\multicolumn{2}{|c|}{$\begin{array}{c}0.05 \\
0.810\end{array}$}} & \multirow{2}{*}{\multicolumn{2}{|c|}{$\begin{array}{c}0.00 \\
0.995\end{array}$}} & \multirow{2}{*}{\multicolumn{2}{|c|}{$\begin{array}{c}3.50^{* *} \\
0.022\end{array}$}} & \multirow{2}{*}{\multicolumn{2}{|c|}{$\begin{array}{c}0.05 \\
0.825\end{array}$}} & \multirow{2}{*}{\multicolumn{2}{|c|}{-0.00}} & \multicolumn{2}{|c|}{$3.39^{* *}$} \\
\hline$P$-value of $t$-test & - & & & & & & & & & & & & \\
\hline
\end{tabular}

\begin{tabular}{|c|c|c|c|c|c|c|c|c|c|c|c|c|}
\hline$c_{s}(\%)$ & $\begin{array}{c}0.33333 \\
\text { T0 } \\
\text { w/o PM }\end{array}$ & $\begin{array}{c}0.33333 \\
\text { T0 } \\
\mathrm{w} / \mathrm{PM}\end{array}$ & $\begin{array}{c}0.33333 \\
\text { UP } \\
\text { w/o PM }\end{array}$ & $\begin{array}{c}0.33333 \\
\text { UP } \\
\text { w/ PM }\end{array}$ & $\begin{array}{c}0.33333 \\
\text { ONS } \\
\text { w/o PM }\end{array}$ & $\begin{array}{c}0.33333 \\
\text { ONS } \\
\mathrm{w} / \mathrm{PM}\end{array}$ & $\begin{array}{c}0.5 \\
\text { T0 } \\
\text { w/o PM }\end{array}$ & $\begin{array}{c}0.5 \\
\text { T0 } \\
\text { w/ PM }\end{array}$ & $\begin{array}{c}0.5 \\
\text { UP } \\
\text { w/o PM }\end{array}$ & $\begin{array}{c}0.5 \\
\text { UP } \\
\mathrm{w} / \mathrm{PM}\end{array}$ & $\begin{array}{c}0.5 \\
\text { ONS } \\
\text { w/o PM }\end{array}$ & $\begin{array}{c}0.5 \\
\text { ONS } \\
\text { w/ PM }\end{array}$ \\
\hline$P$-value of JB test & 0.659 & 0.578 & 0.530 & 0.513 & 0.055 & 0.082 & 0.667 & 0.591 & 0.540 & 0.524 & 0.054 & 0.079 \\
\hline Standard deviation (\%) & 1.53 & 1.53 & 1.50 & 1.50 & 10.08 & 11.11 & 1.52 & 1.53 & 1.50 & 1.50 & 10.06 & 11.06 \\
\hline Mean $(\%)$ & 10.7 & 10.8 & 10.7 & 10.7 & 16.3 & 19.6 & 10.4 & 10.5 & 10.5 & 10.4 & 15.2 & 18.4 \\
\hline Difference of means ${ }^{\mathrm{a}}(\%)$ & $1.37^{* * *}$ & $1.42^{* * *}$ & $1.39^{* * *}$ & $1.39^{* * *}$ & $6.99^{* * *}$ & $10.27^{* * *}$ & $1.09^{* * *}$ & $1.12^{* * *}$ & $1.12^{* * *}$ & $1.11^{* * *}$ & $5.86^{* * *}$ & $9.02^{* * *}$ \\
\hline$P$-value of $t$-test & $5.4 \times 10^{-11}$ & $1.7 \times 10^{-11}$ & $2.2 \times 10^{-11}$ & $2.4 \times 10^{-11}$ & $4.9 \times 10^{-10}$ & $5.5 \times 10^{-15}$ & $1.2 \times 10^{-7}$ & $4.9 \times 10^{-8}$ & $3.9 \times 10^{-8}$ & $4.4 \times 10^{-8}$ & $8.3 \times 10^{-8}$ & $1.2 \times 10^{-12}$ \\
\hline Difference of means ${ }^{\mathrm{b}}(\%)$ & \multirow{2}{*}{\multicolumn{2}{|c|}{0.04}} & \multicolumn{2}{|c|}{-0.00} & \multicolumn{2}{|c|}{$3.28^{* *}$} & \multicolumn{2}{|c|}{0.04} & \multicolumn{2}{|c|}{-0.00} & \multicolumn{2}{|c|}{$3.16^{* *}$} \\
\hline$P$-value of $t$-test & & 0.841 & \multicolumn{2}{|c|}{0.993} & \multicolumn{2}{|c|}{0.030} & \multicolumn{2}{|c|}{0.856} & \multicolumn{2}{|c|}{0.987} & \multicolumn{2}{|c|}{0.036} \\
\hline
\end{tabular}

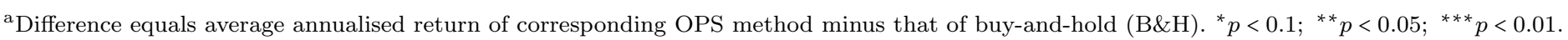

${ }^{\mathrm{b}}$ Difference equals average annualised return of corresponding OPS method with proposed method (PM) minus that without PM. ${ }^{*} p<0.1 ;{ }^{* *} p<0.05 ;{ }^{* * *} p<0.01$.

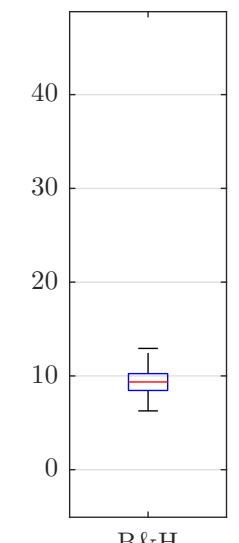

$B \& H$

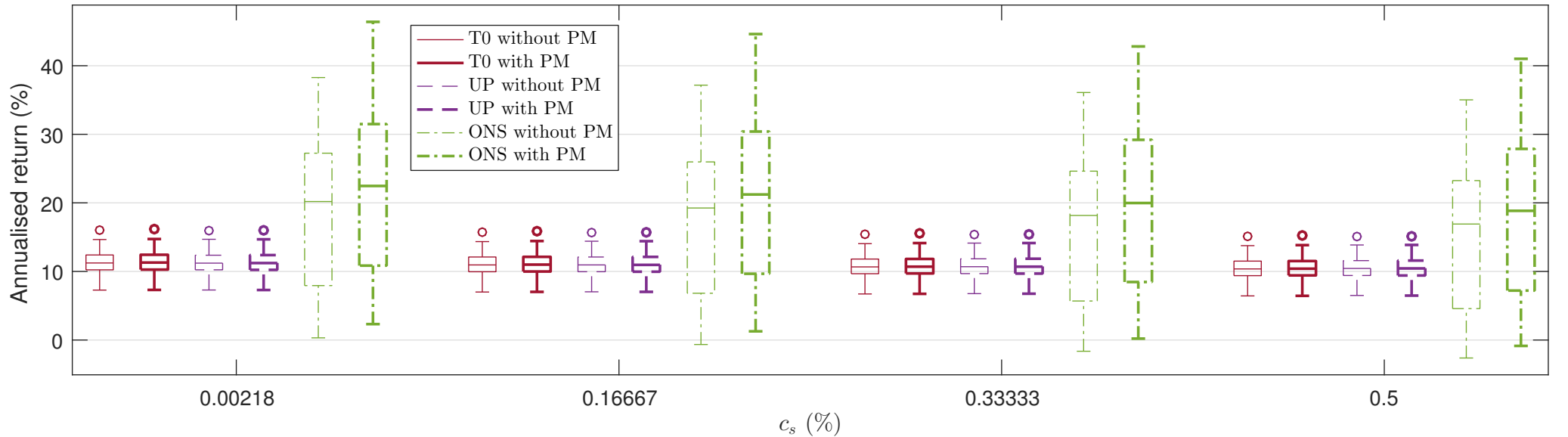

Figure 12. Box plots of annualised returns of different online portfolio selection methods without and with the proposed method $(\mathrm{PM})\left(c_{p}=0, s_{0}=10^{6} \mathrm{USD}\right)$. 


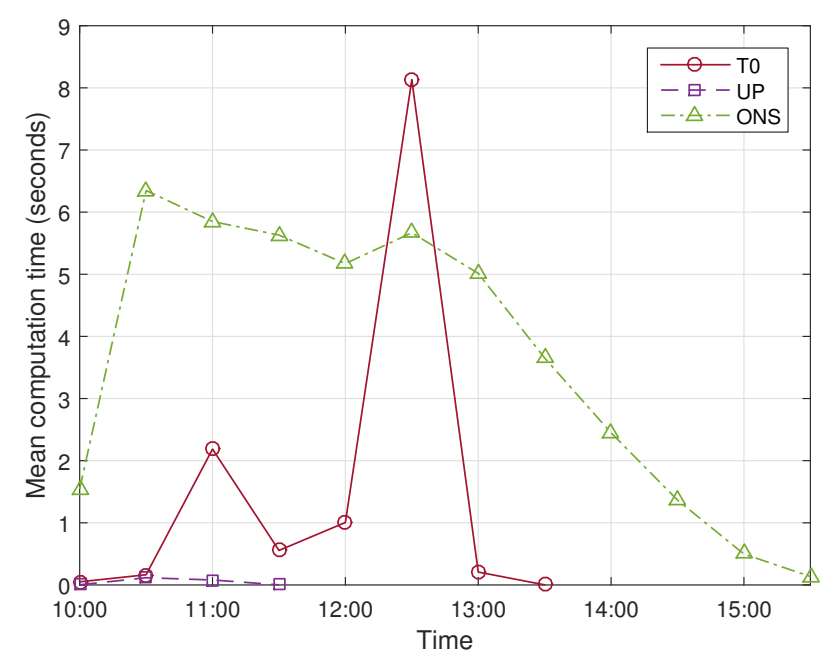

(a) Initial wealth $s_{0}$ is USD 100,000 (the latter parts of T0 and UP are missing as they end intraday trading before 15:30).

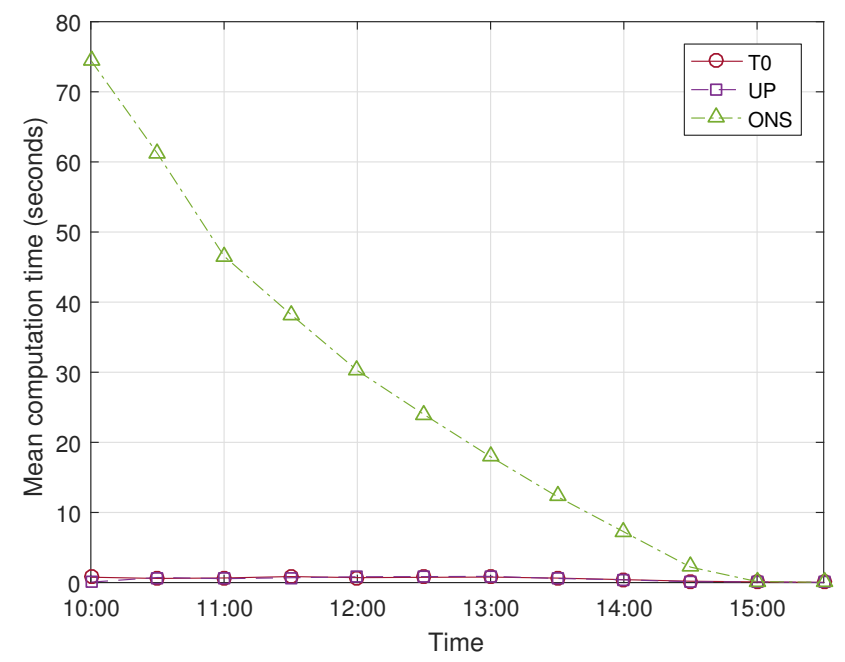

(b) Initial wealth $s_{0}$ is USD $1,000,000$.

Figure 13. The mean computation time of the proposed method (trading at 16:00 is not counted as intraday trading).

\subsection{Computation time}

The mean computation time of the PM depends on an OPS strategy, an initial wealth $s_{0}$, and intraday trading time $t$ as shown in Figure 13. Firstly, the PM requires more computation time for the higher-volume trading algorithms (e.g. ONS) than the lower-volume trading algorithms (e.g. T0 and UP) as shown in both Figure 13(a) and Figure 13(b). Secondly, the PM requires more computation time for the bigger-sized funds (see Figure 13(b)) than the smaller-sized funds (see Figure 13(a)). Thirdly, the computation time changes over time during the day. To be specific, the computation time of the PM with ONS decreases as time goes by during NASDAQ trading hours as shown in Figure 13(b). This is because Algorithm 2 has the iteration for $\left(\tau_{\max }-t\right)$ times for each $t$ (see the 15th line of Algorithm 2) to calculate the minimum optimal number of intraday trades. Consequently, the time complexity of the PM is $O\left(\tau_{\max }\right)$.

\section{Conclusion}

As claimed in the introduction, this paper is, to the best of authors' knowledge, the first attempt to combine OPS with an algorithmic trading under limited market liquidity. We develop a mathematical framework within which the optimal intraday trading strategy minimises overall TCs, consisting of both the proportional TCs and MICs, when rebalancing a multi-asset portfolio.

By considering the real-time LOBs when rebalancing the portfolio, the strategy optimally splits very large market orders into small sequential market orders, which significantly cushions the shock especially for large volume trading. Moreover, the proposed intraday trading algorithm is applicable to any portfolio rebalancing strategy, including all the OPS methods regardless of capital size.

It is also worth noting that the applications of our PM algorithm is not limited to OPS methods mainly discussed in this paper. One could easily apply the PM algorithm to other aspects of financial trading such as optimal control of execution costs of trading a portfolio and .

Using historical NASDAQ LOB data, the numerical experiments and backtesting results have demonstrated that:

- The proposed algorithm is much effective for large capital investment as it generally leads to higher transaction costs;

- Our intraday trading algorithm generates more benefit (higher TCs reduction) for OPS methods 
which rebalance more frequently such as ONS;

- The lower the proportional transaction fees rate is, the more benefit the proposed algorithm creates as the overall TCs is much sensitive to MICs other than the proportional TCs.

The backtesting results are very promising, however, the heavy computation, analysed in Section 6.6, should be reduced further for real-time algorithmic trading.

\section{Acknowledgements}

The financial support of EUR 4,798 from the Department of Economics at the University of Glasgow to purchase the LOBSTER data is acknowledged. In addition, the High Performance Compute Cluster of IT Services at the University of Glasgow reduced the total time required to obtain the MC simulation results in Section 6.

\section{References}

Agarwal, A., Hazan, E., Kale, S. and Schapire, R.E., Algorithms for portfolio management based on the Newton method. In Proceedings of the the 23rd International Conference on Machine Learning, pp. 9--16, 2006.

Agliardi, R. and Gençay, R., Hedging through a limit order book with varying liquidity. Journal of Derivatives, 2014, 22, 32--49.

Alfonsi, A., Fruth, A. and Schied, A., Constrained portfolio liquidation in a limit order book model. In Proceedings of the Advances in mathematics of finance, Vol. 83 of Banach Center publications, pp. 9--25, 2008.

Alfonsi, A., Fruth, A. and Schied, A., Optimal execution strategies in limit order books with general shape functions. Quantitative Finance, 2010, 10, 143--157.

Almgren, R. and Chriss, N., Optimal execution of portfolio transactions. Journal of Risk, 2001, 3, 5--40.

Avellaneda, M. and Lee, J.H., Statistical arbitrage in the US equities market. Quantitative Finance, 2010, 10, 761--782.

Bendtsen, M. and Peña, J.M., Gated Bayesian networks for algorithmic trading. International Journal of Approximate Reasoning, 2016, 69, 58--80.

Bertsimas, D. and Lo, A., Optimal control of execution costs. Journal of Financial Markets, 1998, 1, 1--50.

Birge, J.R. and Louveaux, F., Introduction to stochastic programming (2nd edn), 2011, Springer.

Borodin, A., El-Yaniv, R. and Gogan, V., On the competitive theory and practice of portfolio selection. In LATIN 2000: Theoretical Informatics, Vol. 1776 of Lecture Notes in Computer Science, pp. 173--196, 2000, Springer.

Borodin, A., El-Yaniv, R. and Gogan, V., Can we learn to beat the best stock. Journal of Artificial Intelligence Research, 2004, 21, 579--594.

Bussieck, M.R. and Vigerske, S., MINLP solver software. In Wiley Encyclopedia of Operations Research and Management Science, 2011, Wiley.

Caldeira, J. and Moura, G.V., Selection of a portfolio of pairs based on cointegration: A statistical arbitrage strategy. Brazilian Review of Finance, 2013, 11, 49--80.

Cont, R., Stoikov, S. and Talreja, R., A stochastic model for order book dynamics. Operations Research, 2010, 58, 549--563.

Cover, T.M., Universal portfolios. Mathematical finance, 1991, 1, 1--29.

Crammer, K., Dredze, M. and Pereira, F., Exact convex confidence-weighted learning. In Proceedings of the Advances in Neural Information Processing Systems, 2008.

Damodaran, A., Investment philosophies: Successful strategies and the investors who made them work (2nd edn), 2012, Wiley.

DeMiguel, V., Nogales, F.J. and Uppal, R., Stock return serial dependence and out-of-sample portfolio performance. Review of Financial Studies, 2014, 27, 1031--1073.

Engle, R.F. and Sokalska, M.E., Forecasting intraday volatility in the US equity market. multiplicative component garch. Journal of Financial Econometrics, 2012, 10, 54--83. 
Ferreira, M.A. and Santa-Clara, P., Forecasting stock market returns: The sum of the parts is more than the whole. Journal of Financial Economics, 2011, 100, 514--537.

Freitas, F.D., De Souza, A.F. and de Almeida, A.R., Prediction-based portfolio optimization model using neural networks. Neurocomputing, 2009, 72, 2155--2170.

Guéant, O., Lehalle, C.A. and Fernandez-Tapia, J., Optimal portfolio liquidation with limit orders. SIAM Journal on Financial Mathematics, 2012, 3, 740--764.

Györfi, L., Lugosi, G. and Udina, F., Nonparametric kernel-based sequential investment strategies. Mathematical Finance, 2006, 16, 337--357.

Györfi, L., Udina, F. and Walk, H., Nonparametric nearest neighbor based empirical portfolio selection strategies. Statistics \& Decisions, 2008, 26, 145--157.

Györfi, L. and Vajda, I., Growth optimal investment with transaction costs. In Algorithmic Learning Theory, pp. 108--122, 2008, Springer.

Ha, Y. and Zhang, H., Liquidity Risks, Transaction costs and Online Portfolio Selection. Working paper, 2018.

Helmbold, D.P., Schapire, R.E., Singer, Y. and Warmuth, M.K., On-line portfolio selection using multiplicative updates. Mathematical Finance, 1998, 8, 325--347.

Huang, R. and Polak, T., LOBSTER: Limit order book reconstruction system. Working paper, available at http://ssrn. com/abstract=1977207, 2011.

Ishijima, H., Numerical methods for universal portfolios. In Proceedings of the Quantitative Methods in Finance Conference, 2001.

Kissell, R., The science of algorithmic trading and portfolio management, 2013, Academic Press.

Kissell, R., Glantz, M. and Malamut, R., Optimal trading strategies: Quantitative approaches for managing market impact and trading risk, 2003, AMACOM.

Kissell, R., Glantz, M. and Malamut, R., A practical framework for estimating transaction costs and developing optimal trading strategies to achieve best execution. Finance Research Letters, 2004, 1, 35--46.

Kozat, S.S. and Singer, A.C., Universal semiconstant rebalanced portfolios. Mathematical Finance, 2011, 21, 293--311.

Krauss, C., Do, X.A. and Huck, N., Deep neural networks, gradient-boosted trees, random forests: Statistical arbitrage on the S\&P 500. European Journal of Operational Research, 2017, 259, 689--702.

Li, B. and Hoi, S.C., On-line portfolio selection with moving average reversion. In Proceedings of the 29th International Conference on Machine Learning, 2012.

Li, B., Hoi, S.C. and Gopalkrishnan, V., Corn: Correlation-driven nonparametric learning approach for portfolio selection. ACM Transactions on Intelligent Systems and Technology, 2011, 2.

Li, B., Hoi, S.C., Zhao, P. and Gopalkrishnan, V., Confidence weighted mean reversion strategy for online portfolio selection. ACM Transactions on Knowledge Discovery from Data, 2013, 7, 4.

Li, B. and Hoi, S.C.H., Online portfolio selection: Principles and algorithms, 2015, CRC Press.

Li, B., Zhao, P., Hoi, S.C. and Gopalkrishnan, V., PAMR: Passive aggressive mean reversion strategy for portfolio selection. Machine Learning, 2012, 87, 221--258.

Lorenz, J.M., Optimal trading algorithms: Portfolio transactions, multiperiod portfolio selection, and competitive online search. PhD thesis, ETH Zurich, 2008.

Matheson, T., Taxing financial transactions: Issues and evidence. Working paper 11/54, International Monetary Fund, 2011.

Mousavi, S., Esfahanipour, A. and Zarandi, M.H.F., A novel approach to dynamic portfolio trading system using multitree genetic programming. Knowledge-Based Systems, 2014, 66, 68--81.

Olsson, R., Portfolio management under transaction costs: Model development and Swedish evidence. PhD thesis, Umeå University, 2005.

Otranto, E., Asset allocation using flexible dynamic correlation models with regime switching. Quantitative Finance, 2010, 10, 325--338.

Palczewski, J., Poulsen, R., Schenk-Hoppé, K.R. and Wang, H., Dynamic portfolio optimization with transaction costs and state-dependent drift. European Journal of Operational Research, 2015, 243, 921--931.

Simon, D., Evolutionary optimization algorithms, 2013, Wiley.

Tan, Z., Quek, C. and Cheng, P.Y., Stock trading with cycles: A financial application of ANFIS and reinforcement learning. Expert Systems with Applications, 2011, 38, 4741--4755.

Xu, H.C., Chen, W., Xiong, X., Zhang, W., Zhou, W.X. and Stanley, H.E., Limit-order book resiliency after effective market orders: Spread, depth and intensity. Journal of Statistical Mechanics: Theory and 


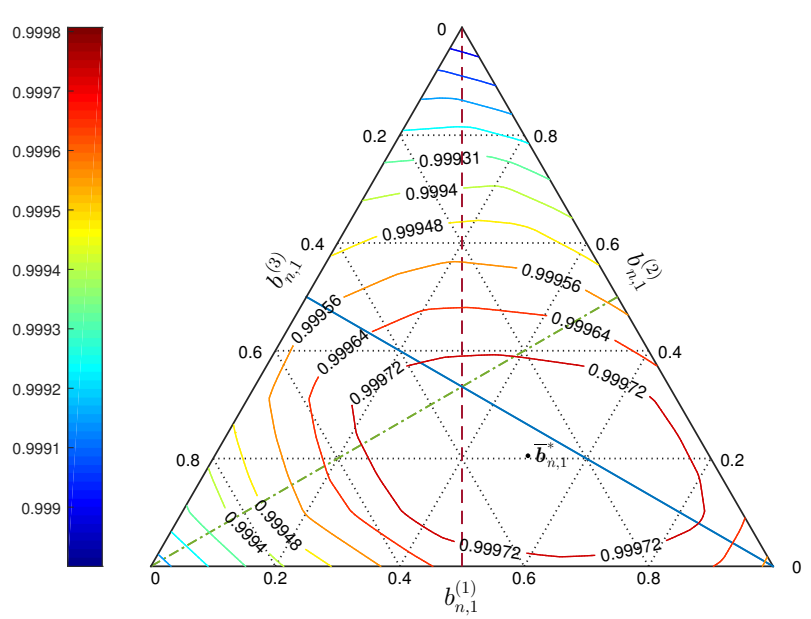

(a) Ternary contour plot of $w_{n} \bar{w}_{n, 1}$.

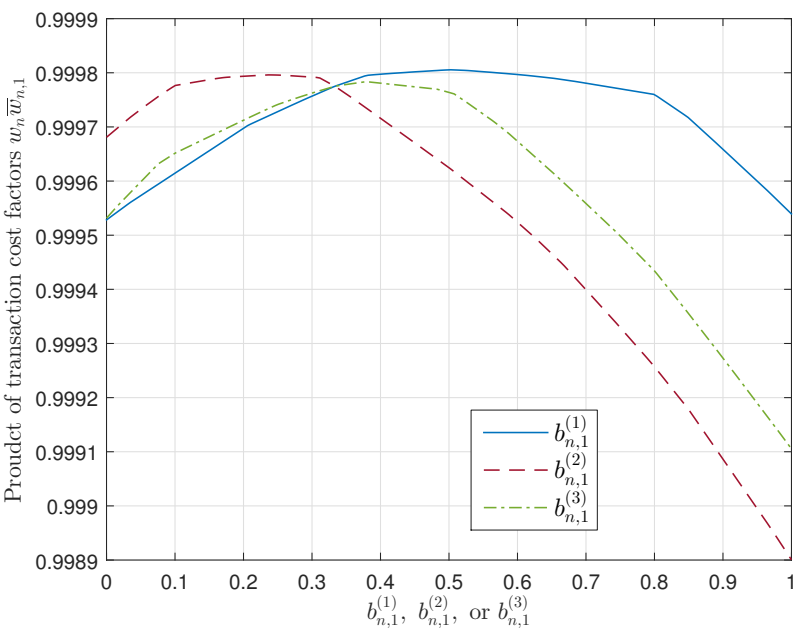

(b) $1 \mathrm{D}$ plots of $w_{n} \bar{w}_{n, 1}$ (each line matches each straight line in the left plot).

Figure A1. Ternary contour plots and 1D plots of the product of transaction cost factors $w_{n} \bar{w}_{n, 1}$ with the variable $\boldsymbol{b}_{n, 1}$ and the fixed values: $\boldsymbol{b}_{n}=\left[\begin{array}{lll}1 / 3 & 1 / 3 & 1 / 3\end{array}\right]^{\mathrm{T}}, \boldsymbol{x}_{n, 0}=\left[\begin{array}{lll}0.6 & 0.9 & 1.4\end{array}\right]^{\mathrm{T}}, \nu_{n-1, \tau}=10^{6} \mathrm{USD}, \boldsymbol{b}_{n+1}=\left[\begin{array}{lll}0.8 & 0.8 & 0.1\end{array}\right]^{\mathrm{T}}, c_{p}=0$, and $c_{s}=0.00218 \%$. 10-level limit order book data of AAPL $\left(b^{(1)}\right)$, AMZN $\left(b^{(2)}\right)$, and GOOG $\left(b^{(3)}\right)$ on 21 Jun 2012 at 16:00:00 was used.

Experiment, 2017.

\section{Appendix A: An example of unimodality of $w_{n} \bar{w}_{n, 1}$}

The product of TCFs $w_{n} \bar{w}_{n, 1}$ in Equation (22) can be rewritten as a function of $\boldsymbol{b}_{n, 1}$ as

$$
\begin{aligned}
w_{n} \bar{w}_{n, 1} & =w\left(\boldsymbol{b}_{n}, \boldsymbol{b}_{n, 1}, \boldsymbol{x}_{n, 0}, \nu_{n-1, \tau}\right) w\left(\boldsymbol{b}_{n, 1}, \boldsymbol{b}_{n+1}, \mathbf{1}, s_{n} w_{n}\right) \\
& =w\left(\boldsymbol{b}_{n}, \boldsymbol{b}_{n, 1}, \boldsymbol{x}_{n, 0}, \nu_{n-1, \tau}\right) w\left(\boldsymbol{b}_{n, 1}, \boldsymbol{b}_{n+1}, \mathbf{1}, \nu_{n-1, \tau}\left\langle\boldsymbol{b}_{n}, \boldsymbol{x}_{n, 0}\right\rangle w_{n}\right) \\
& =w\left(\boldsymbol{b}_{n}, \boldsymbol{b}_{n, 1}, \boldsymbol{x}_{n, 0}, \nu_{n-1, \tau}\right) w\left(\boldsymbol{b}_{n, 1}, \boldsymbol{b}_{n+1}, \mathbf{1}, \nu_{n-1, \tau}\left\langle\boldsymbol{b}_{n}, \boldsymbol{x}_{n, 0}\right\rangle w\left(\boldsymbol{b}_{n}, \boldsymbol{b}_{n, 1}, \boldsymbol{x}_{n, 0}, \nu_{n-1, \tau}\right)\right),
\end{aligned}
$$

and it is plotted as a unimodal function of $\boldsymbol{b}_{n, 1}$ (i.e. $w_{n} \bar{w}_{n, 1}$ strictly decreases as $\boldsymbol{b}_{n, 1}$ goes away from the maximum point $\overline{\boldsymbol{b}}_{n, 1}^{*}$ ) as shown in Figure A1. Therefore, $w_{n} \bar{w}_{n, 1}$ in Figure A1 is a unimodal function of $\boldsymbol{b}_{n, 1} \in \Delta^{2}$. However, this is only one example from the given values $\left(\boldsymbol{b}_{n}, \boldsymbol{x}_{n, 0}, \nu_{n-1, \tau}\right.$, $\boldsymbol{b}_{n+1}, c_{p}$, and $c_{s}$ ) and the LOBs of the three stocks. The mathematical proof of the unimodality of $w_{n} \bar{w}_{n, 1}$ is not provided in this paper. 